\title{
Hydrodynamic Limit for a System with Finite Range Interactions
}

\section{Fraydoun Rezakhanlou}

Courant Institute of Mathematical Sciences, New York University, 251 Mercer Street, New York, NY 10012, USA

\begin{abstract}
We study a system of interacting diffusions. The variables present the amount of charge at various sites of a periodic multidimensional lattice. The equilibrium states of the diffusion are canonical Gibbs measures of a given finite range interaction. Under an appropriate scaling of lattice spacing and time, we derive the hydrodynamic limit for the evolution of the macroscopic charge density.
\end{abstract}

\section{Introduction}

The derivation of the hydrodynamic equation for infinite particle systems with conservation law has been the subject of active research. One such model is the Ginzburg-Landau model [11]. The hydrodynamic equation for this model is obtained in [3] and [4]. In this model charges are located at the various sites of a periodic multidimensional lattice. The flow of these charges from one site to another is governed by a suitable diffusion law. After an appropriate space and time scaling, the microscopic charge density converges to a deterministic limit which is characterized as the solution of a nonlinear parabolic equation.

The passage to the hydrodynamic limit for the Ginzburg-Landau model under certain conditions was studied in [4]. We describe these conditions.

For any positive integer $N$, let $S_{N}$ denote the periodic lattice $\{j: j=0,1, \ldots, N\}$ with 0 and $N$ identified, and let $S_{N}^{d}$ denote the product of $d$ copies of $S_{N}$. For each site $a$ in $S_{N}^{d}$, there is a random variable $x_{a}=x_{a}(t)$ which is the amount of charge at site $a$. The family of $x_{a}$ undergo a diffusion with generator

$$
\mathscr{L}_{N}^{0}=\frac{N^{2}}{2}\left[\sum\left(\frac{\partial}{\partial x_{a}}-\frac{\partial}{\partial x_{b}}\right)^{2}-\sum\left(\phi^{\prime}\left(x_{a}\right)-\phi^{\prime}\left(x_{b}\right)\right)\left(\frac{\partial}{\partial x_{a}}-\frac{\partial}{\partial x_{b}}\right)\right]
$$

where both sums are over the adjacent sites $a$ and $b$ in $S_{N}^{d}$. The generator $\mathscr{L}_{N}^{0}$ is formally symmetric with respect to the product measure $\rho_{N}(d \underline{x})$ defined as

$$
\rho_{N}(d \underline{x})=\prod_{a \in S_{N}^{d}} e^{-\phi\left(x_{a}\right)} d x_{a},
$$


where $\underline{x}$ denotes the vector $\left(x_{a} ; a \in S_{N}^{d}\right)$. The function $\phi: \mathbf{R} \rightarrow \mathbf{R}$ is a continuously differentiable function with the following properties.

$$
\begin{aligned}
\int e^{-\phi(x)} d x & =1, \\
\int e^{\lambda x-\phi(x)} d x & <\infty, \\
\int e^{\lambda\left|\phi^{\prime}(x)\right|-\phi(x)} d x & <\infty
\end{aligned}
$$

for all $\lambda$ in $\mathbf{R}$.

The diffusion generated by $\mathscr{L}_{N}^{0}$ starts from an initial distribution which has a density $f_{N}^{0}$ with respect to the measure $\rho_{N}$ and $f_{N}^{0}$ satisfies the entropy bound

$$
\frac{1}{N^{d}} \int f_{N}^{0} \log f_{N}^{0} d \rho_{N} \leqq C
$$

uniformly in $N$ and for some finite constant $C$. Then the evolution corresponding to the diffusion will give us a density $f_{N}^{t}$ which satisfies the forward equation,

$$
\frac{\partial f_{N}^{t}}{\partial t}=\mathscr{L}_{N}^{0} f_{N}^{t} .
$$

Associated with the charge configuration $\underline{x} \in \mathbf{R}^{N^{d}}$ and $t \geqq 0$, we define the signed measure

$$
\mu_{N}(t)=\frac{1}{N^{d}} \sum_{a \in S_{N}^{d}} x_{a}(t) \delta_{a / N} .
$$

Let $S$ be the unit circle or the interval $0 \leqq \theta \leqq 1$ with 0 and 1 identified, and let $S^{d}$ be the product of $d$ copies of $S$. Then $\mu_{N}(t)=\mu_{N}(t, d \theta)$ is a measure on the $d$-dimensional torus $S^{d}$.

We assume that for some nice function $m_{0}(\theta)$ and any positive $\delta$

$$
\lim _{N \rightarrow \infty} \int_{E_{N, \delta}} f_{N}^{0} d \rho_{N}=0,
$$

where

$$
E_{N, \delta}=\left\{\underline{x}:\left|\frac{1}{N^{d}} \sum J(a / N) x_{a}-\int J(\theta) m_{0}(\theta) d \theta\right| \geqq \delta\right\} .
$$

Here $d \theta$ denotes the normalized Haar measure on $S^{d}$. It is shown in [4] that there exists a function $m(t, \theta)$ such that

$$
\lim _{N \rightarrow \infty} \int J(\theta) \mu_{N}(t, d \theta)=\int J(\theta) m(t, \theta) d \theta,
$$

and this function satisfies a nonlinear parabolic equation which will be described below (see (1.13)).

We generalize the above result by adding an interaction term to the function $-\phi(x)$ in the measure $\rho_{N}$. This means that we replace the product measure $\rho_{N}$ with a Gibbs measure which has an interaction of finite range. The important aspect of our work is that we do not exclude the occurrence of phase transition in the space of Gibbs measures. 
Let $F$ be a function which depends on a fixed finite number of coordinates. We assume that $F$ is bounded, continuously differentiable and has bounded first derivatives. The interaction energy corresponding to the interaction field $F$ is defined by

$$
\mathscr{H}_{N}(\underline{x})=\sum_{a \in S_{N}^{d}} F\left(\tau_{a} \underline{x}\right)
$$

where $\tau_{a}$ denotes the shift operator in the space $S_{N}^{d}$. We now replace the generator $\mathscr{L}_{N}^{0}$ with

$$
\begin{aligned}
\mathscr{L}_{N}= & \frac{N^{2}}{2}\left[\sum\left(\frac{\partial}{\partial x_{a}}-\frac{\partial}{\partial x_{b}}\right)^{2}\right. \\
& \left.-\sum\left(\phi^{\prime}\left(x_{a}\right)-\phi^{\prime}\left(x_{b}\right)+\frac{\partial \mathscr{H}_{N}}{\partial x_{b}}(\underline{x})-\frac{\partial \mathscr{H}_{N}}{\partial x_{a}}(\underline{x})\right)\left(\frac{\partial}{\partial x_{a}}-\frac{\partial}{\partial x_{b}}\right)\right] .
\end{aligned}
$$

This generator is reversible with respect to the Gibbs measure $v_{N}$ defined as

$$
v_{N}(d \underline{x})=\frac{1}{Z_{N}} \exp \left(\mathscr{H}_{N}(\underline{x})\right) \rho_{N}(d \underline{x}),
$$

where $Z_{N}$ is the normalizing constant.

We now assume that the diffusion generated by $L_{N}$ starts from an initial distribution which has a density $f_{N}^{0}$ with respect to the measure $v_{N}$ and $f_{N}^{0}$ satisfies the entropy bound

$$
\frac{1}{N^{d}} \int f_{N}^{0} \log f_{N}^{0} d v_{N} \leqq C
$$

uniformly in $N$ and for some finite constant $C$. Then the evolution corresponding to the diffusion will give us a density $f_{N}^{t}$ which satisfies

$$
\frac{\partial f_{N}^{t}}{\partial t}=\mathscr{L}_{N} f_{N}^{t}
$$

We also assume that for some nice function $m_{0}(\theta)$ and any positive $\delta$,

where

$$
\lim _{N \rightarrow \infty} \int_{E_{N, \delta}^{0}} f_{N}^{0} d v_{N}=0
$$

$$
E_{N, \delta}^{0}=\left\{\underline{x}:\left|\frac{1}{N^{d}} \sum J(a / N) x_{a}-\int J(\theta) m_{0}(\theta) d \theta\right| \geqq \delta\right\} .
$$

We define $h$ as the convex conjugate of the specific free energy $\varphi$ defined by

$$
\varphi(\lambda)=\lim _{N \rightarrow \infty} \frac{1}{N^{d}} \log \int \exp \left(\lambda \sum_{a} x_{a}\right) v_{N}(d \underline{x}) .
$$

We now state our main result.

Theorem 1.1. If the initial distribution of charges satisfies (1.6') and (1.9'), then for 
all time $t$, every smooth $J$ and each $\delta>0$,

where

$$
\lim _{N \rightarrow \infty} \int_{E_{N, \delta}^{t}} f_{N}^{t} d v_{N}=0
$$

$$
E_{N, \delta}^{t}=\left\{\underline{x}:\left|\frac{1}{N^{d}} \sum J(a / N) x_{a}-\int J(\theta) m(t, \theta) d \theta\right| \geqq \delta\right\},
$$

and $m(t, \theta)$ satisfies weakly the following nonlinear parabolic equation:

$$
\frac{\partial m}{\partial t}=\frac{1}{2} \Delta h^{\prime}(m(t, \theta)), \quad m(0, \theta)=m_{0}(\theta) .
$$

When $F=0$, then $h^{\prime}$ is strictly increasing. It is well known in statistical mechanics that for some nonzero $F$, the function $h^{\prime}$ is not strictly increasing, which makes the evolution Eq. (1.13) degenerate. This corresponds to a phase transition in physics. Nonetheless we can still derive the hydrodynamic equation for the charge density $m$. We will also verify properties such as uniqueness of solutions, maximum principle and the asymptotic behavior of the solution $m(t, \theta)$ as time goes to infinity.

In the presence of phase transition, our result implies that the different phases do not segregate macroscopically at the hydrodynamic time scale. This is consistent with the Lifshitz-Slyozov theory. According to this theory, it takes times of the order $N^{3}$ for forming a macroscopic droplet. Therefore segregation cannot be seen on the hydrodynamic time scale.

Let $J$ be a fixed smooth function and consider the stochastic process

$$
y_{N}(t)=\int J(\theta) \mu_{N}(t, d \theta)=\frac{1}{N^{d}} \sum J(a / N) x_{a}(t) .
$$

It is not hard to see that

$$
d y_{N}(t)=d M_{N}(t)+A_{N}(t) d t,
$$

where $M_{N}(t)$ is a continuous martingale which goes to zero as $N$ goes to infinity, and

$$
\begin{aligned}
A_{N}(t) & \approx \frac{1}{2 N^{d}} \sum \Delta J(a / N) G_{0}\left(\tau_{a} \underline{x}(t)\right), \\
G_{0}(\underline{x}) & =\phi^{\prime}\left(x_{0}\right)-\frac{\partial \mathscr{H}_{N}}{\partial x_{0}}(\underline{x}) .
\end{aligned}
$$

The main step in the proof of the hydrodynamic behavior for our example (like [4]) is that

$$
\lim _{N \rightarrow \infty} \int_{0}^{t} A_{N}(s) d s=\frac{1}{2} \int_{0}^{t} \int \Delta J(\theta) h^{\prime}(m(\theta, s)) d s d \theta,
$$

where $m$ is the solution of (1.13).

When $F=0$, then for any bounded continuous local function $G$ (i.e. depending on a fixed finite number of coordinates) and for any smooth test function $J$, it is 
shown in [4] that

$$
\lim _{N \rightarrow \infty} \frac{1}{N^{d}} \sum_{a} J(a / N) \int_{0}^{t} G\left(\tau_{a} \underline{x}(s)\right) d s=\int_{0}^{t} \int J(\theta) B_{G}(m(s, \theta)) d s d \theta,
$$

where

$$
\begin{aligned}
B_{G}(y) & =\int G(\underline{x}) \prod_{a} \frac{1}{M(\lambda)} e^{\lambda x_{a}-\phi\left(x_{a}\right)} d x_{a}, \\
M(\lambda) & =\int e^{\lambda x-\phi(x)} d x
\end{aligned}
$$

and

$$
\lambda=h^{\prime}(y)
$$

In general, if $F \neq 0$ and if phase transition occurs, we do not expect to have (1.16) for any $G$. We can however prove (1.16) for $G=G_{0}$ and obtain (1.15). This is because of a very special form of $G_{0}$ which makes it possible to avoid any problem coming from phase transitions.

We expect that the distribution of the charge configuration $\underline{x}(t)$ for $t>0$, looks somewhat like

$$
c_{N} \exp \left(\sum_{a} \lambda_{a} x_{a}\right) d v_{N},
$$

where $\lambda_{a} \approx h^{\prime}(m(t, a / N))$. As in [4], we use the entropy production as a technical tool to prove the validity of such a picture. This is the content of Sect. 3. Before this, we need to establish a local ergodic theorem. This is done in Sect. 2. In this section, we basically use the techniques of large deviations. Finally in Sect. 5 we derive the hydrodynamic equation and study some of its properties.

\section{Large Deviations for Conditioned Gibbs Measures}

The object of this section is a large deviations result (and its corollaries) for the conditioned Gibbs measures. First we start with some preliminary considerations related to theory of the large deviations. Let $X$ be a Polish space with Borel field $\mathscr{B}$. A function $I: X \rightarrow[0, \infty]$ is called a rate function if it is lower semicontinuous and the set $\{x \mid I(x) \leqq \ell\}$ is compact for all finite $\ell$. A family $P_{n}$ of probability measures on $X$ is said to have the large deviation property with rate $I$ if there exists a sequence $a_{n}$ which tends to $\infty$ and

$$
\limsup _{n \rightarrow \infty} \frac{1}{a_{n}} \log \mu_{n}(C) \leqq-\inf _{x \in C} I(x)
$$

for all closed subsets $C$ of $X$, and

$$
\liminf _{n \rightarrow \infty} \frac{1}{a_{n}} \log \mu_{n}(O) \geqq-\inf _{x \in O} I(x)
$$

for all open subsets $O$ of $X$.

Our first lemma is a useful form of the so-called Contraction Principle. 
Lemma 2.1. Let $\Phi: X_{0} \rightarrow Y$, where $X_{0}$ is a subset of $X$ and $Y$ is another Polish space. Suppose that for each positive number $\ell$ there exists a closed subset $C_{\ell}$ of $X$ such that $\Phi$ restricted to $C_{\ell}$ is continuous and moreover

$$
\limsup _{\ell \rightarrow \infty} \limsup _{n \rightarrow \infty} \frac{1}{a_{n}} \log P_{n}\left(X-C_{\ell}\right)=-\infty \text {. }
$$

Then the family $P_{n} \circ \Phi^{-1}$ has the large deviation property with the rate $\hat{I}(x)=$ $\inf _{\varphi(x)=y} I(x)$.

Proof. Let $\hat{C}$ be any closed subset of $Y$. Then

$$
P_{n}(\Phi \in \hat{C}) \leqq P_{n}\left(\{\Phi \in \hat{C}\} \cap C_{\ell}\right)+P_{n}\left(X-C_{\ell}\right) .
$$

The set $C=\{\Phi \in \hat{C}\} \cap C_{\ell}$ is closed and

$$
\limsup _{n \rightarrow \infty} \frac{1}{a_{n}} \log P_{n}\left(\{\Phi \in \hat{C}\} \cap C_{\ell}\right) \leqq-\inf _{x \in C} I(x) \leqq-\inf _{y \in \hat{C}} \hat{I}(y) .
$$

So, we have the upper bound estimate because $P_{n}\left(X-C_{\ell}\right)$ is superexponentially small when $l \rightarrow \infty$.

For the lower bound estimate, let $\hat{O}$ be an open subset of $Y$,

$$
P_{n}(\Phi \in \hat{O}) \geqq P_{n}\left(\{\Phi \in \hat{O}\} \cup\left(X-C_{\ell}\right)\right)-P_{n}\left(X-C_{\ell}\right) .
$$

The set $O=\{\Phi \in \hat{O}\} \cup\left(X-C_{\ell}\right)$ is open and

$$
\liminf _{n \rightarrow \infty} \frac{1}{a_{n}} \log P_{n}\left(\{\Phi \in \hat{O}\} \cup\left(X-C_{\ell}\right)\right) \geqq-\inf _{x \in O} I(x) \geqq-\inf _{y \in \hat{O}} \hat{I}(y) .
$$

Moreover $P_{n}\left(X-C_{\ell}\right)$ is superexponentially small as $l \rightarrow \infty$.

It is not hard to check that $\hat{I}$ is a rate function. We omit the proof of it.

We recall the following result which can be found in [2].

Lemma 2.2. Let $W_{n}$ be a sequence of $\mathbf{R}^{d}$-valued random variables on a probability space $\left(\Omega_{n}, \mathscr{F}_{n}, Q_{n}\right)$. If

$$
c(t)=\lim _{n \rightarrow \infty} \frac{1}{a_{n}} \log E^{Q_{n}} e^{t \cdot W_{n}}
$$

exists for all $t$ in $\mathbf{R}^{d}$, then the family

$$
P_{n}(d x)=Q_{n}\left(\frac{W_{n}}{a_{n}} \in d x\right)
$$

has the upper bound large deviation property with the rate $I(t)$ that is the convex conjugate of $c(t)$.

Lemma 2.3. Suppose $\mathscr{A}$ is a collection of closed subsets of $X$ such that for any closed subset $C$ of $X$, there exists a decreasing sequence $A_{m}$ in $\mathscr{A}$ satisfying $\bigcap_{m} A_{m}=C$. If (2.1) holds for all $A$ in $\mathscr{A}$, then it holds for all closed sets $C$.

Proof. Suppose that (2.1) holds for all $A$ in $\mathscr{A}$. Let $C$ be a closed subset of $X$ and 
$C=\bigcap_{m} A_{m}$ for some decreasing subsequence $A_{m}$ of $\mathscr{A}$. Then

$$
\limsup _{n \rightarrow \infty} \frac{1}{a_{n}} \log P_{n}(C) \leqq \inf _{m} \limsup \log P_{n}\left(A_{m}\right) \leqq-\sup _{m} \inf _{x \in A_{m}} I(x) .
$$

We will show

$$
\inf _{x \in C} I(x) \leqq \sup _{m} \inf _{x \in A_{m}} I(x) .
$$

Suppose that $\sup _{m} \inf _{x \in A_{m}} I(x)$ is finite. Set $K_{l}=\{x \mid I(x) \leqq l\}$, where

$$
l=\sup _{m} \inf _{x \in A_{m}} I(x)+1 .
$$

Then $\inf _{x \in A_{m}} I(x)=\inf _{x \in A_{m} \cap K_{l}} I(x)$ and therefore there exists $x_{m} \in A_{m} \cap K_{l}$ such that

$$
\inf _{x \in A_{m}} I(x)=I\left(x_{m}\right)
$$

We may choose a subsequence of $x_{m}$ which converges to some $y \in \bigcap_{m} A_{m}=C$. Therefore

$$
\sup _{m} \inf _{x \in A_{m}} I(x) \geqq \liminf _{m \rightarrow \infty} I\left(x_{m}\right) \geqq I(y) \geqq \inf _{x \in C} I(x) .
$$

Before we state the main result of this section, let us recall the definition and some properties of Gibbs measures.

We consider the configuration space $X=\mathbf{R}^{\mathbb{Z}^{d}}$ which is the set of $x: \mathbb{Z}^{d} \rightarrow \mathbf{R}$ or $\underline{x}=\left(x_{a} ; a \in \mathbb{Z}^{d}\right)$. The set $X$ is endowed with the product topology which makes $X$ a Polish space. For $a$, let $\tau_{a}$ be the translation operator on $X$ defined by $\left(\tau_{a} \underline{x}\right)(b)=$ $\underline{x}(a+b) . \mathscr{M}(X)$ denotes the space of probability measures and $\mathscr{M}_{\tau}(X)$ denotes the space of translation invariant probability measures on $X$. Both of these spaces are endowed with the topology of weak convergence.

For each $n \in \mathbb{Z}^{+}$, we define

$$
T_{n}=\left\{a \in \mathbb{Z}^{d} \mid 0 \leqq a_{j} \leqq n \text { for all } j, \text { where } a=\left(a_{j}: 1 \leqq j \leqq d\right)\right\} .
$$

For each $x \in X$ and $n \in \mathbb{Z}^{+}$, we define $\underline{x}^{n}$ by

$$
\begin{gathered}
\underline{x}^{n}(a)=\underline{x}(a) a \in T_{n}, \\
\underline{x}^{n}\left(a+(n+1) \mathbf{e}^{j}\right)=\underline{x}^{n}(a) ; a \in \mathbb{Z}^{d},
\end{gathered}
$$

where $\mathbf{e}_{k}^{j}=\delta_{j, k} . X_{n}$ denotes the set of all $\underline{x}^{n}$. The empirical process is defined as

$$
R_{n, \underline{x}}=\left|T_{n}\right|^{-1} \sum_{a \in T_{n}} \delta_{\tau_{a} \underline{x^{n}}}
$$

where $\left|T_{n}\right|$ is the number of sites in $T_{n}$. It is easy to see that $R_{n, \underline{x}} \in \mathscr{M}_{\tau}(X)$.

Let $\phi: \mathbf{R} \rightarrow \mathbf{R}$ be a continuously differentiable function satisfying

$$
\begin{array}{r}
\int e^{-\phi(x)} d x=1, \\
\int e^{\lambda x-\phi(x)} d x<\infty, \\
\int e^{\lambda\left|\phi^{\prime}(x)\right|-\phi(x)} d x<\infty
\end{array}
$$


for all real $\lambda$. Equations (2.4) and (2.5) are equivalent to saying that there exists a function $w(x)$ which is symmetric and convex on $\mathbf{R}$ with

$$
\begin{aligned}
& \lim _{x \rightarrow \infty} \frac{|x|}{w(x)}=0, \quad w(x) \geqq|x| \quad \text { for all } x \text { and } \\
& \int e^{w(x)-\phi(x)} d x<\infty, \quad \int e^{w\left(\phi^{\prime}(x)\right)-\phi(x)} d x<\infty .
\end{aligned}
$$

We consider the following classes of local functions:

$C_{\mathrm{loc}}^{0}=\{G: X \rightarrow \mathbf{R}$ such that $G$ is bounded continuous and depends on a fixed finite number of coordinates $\}$,

$C_{\mathrm{loc}}^{1}=\left\{G \in C_{\mathrm{loc}}^{0}: G\right.$ is continuously differentiable with bounded first derivatives $\}$, $C_{\mathrm{loc}}^{\infty}=\left\{G \in C_{\mathrm{loc}}^{0}: G\right.$ is smooth with bounded derivatives $\}$.

Let $\underline{x} \in X$ and $T$ be a subset of $\mathbb{Z}^{d}$. Then $\underline{x}_{T}$ denotes the restriction of $\underline{x}$ to $T$. That is $\underline{x}_{T}: T \rightarrow \mathbf{R}$ and $\underline{x}_{T}(a)=\underline{x}(a)$ for $a \in T$. If $\underline{z}$ is another configuration, then $\underline{x}_{T} \vee \underline{z}$ denotes the configuration which agrees with $\underline{x}$ on $T$ and with $\underline{z}$ on $T^{c}=\mathbb{Z}^{d}-T$. In other words $\underline{x}_{T} \vee \underline{z}$ is the configuration $\underline{x}$ on $T$ with the boundary condition $\underline{z}$.

Let $F$ be a function in $C_{\mathrm{loc}}^{0}$ which depends only on the coordinates $x_{a}$ for $a$ in a finite set $\Lambda \subset \mathbb{Z}^{d}$. We define the interaction energy by

$$
\mathscr{H}_{\boldsymbol{F}, \boldsymbol{T}}(\underline{x})=\sum F\left(\tau_{a} \underline{x}\right)
$$

where the sum is over indices $a$ such that $a+\Lambda \leqq T$. Given a boundary condition $\underline{z}$ in $X$, we define

$$
\mathscr{H}_{F, T, \underline{z}}(\underline{x})=\mathscr{H}_{F, T}(\underline{x})+\sum F\left(\left(\tau_{a} \underline{x}\right)_{T} \vee \underline{z}\right)
$$

where the sum is over $a$ such that

$$
(a+\Lambda) \cap T \neq \varnothing \quad \text { and } \quad(a+\Lambda) \cap T^{c} \neq \varnothing .
$$

We also define the interaction energy for a configuration with periodic boundary conditions. For this, one considers the space of periodic configurations $X_{n}$ and defines

$$
\hat{\mathscr{H}}_{F, n}(\underline{x})=\sum_{a \in S_{n}} F\left(\tau_{a} \underline{x}^{n}\right),
$$

where $S_{n}$ is obtained by identifying the opposite faces of $T_{n}$.

We take the probability measure $\rho(d x)=e^{-\phi(x)} d x$ and for each subset $T$ of $\mathbb{Z}^{d}$, the measure $\rho^{T}$ is the product measure of $\rho$ which is defined on $\mathbf{R}^{T}$. Now we define the finite volume Gibbs measure $v_{F, T}$ associated with $F$ and $\rho$ by

$$
v_{F, T}(d \underline{x})=\frac{1}{Z_{F, T}} \exp \left(\mathscr{H}_{F, T}(\underline{x})\right) \rho^{T}(d \underline{x}),
$$

where

$$
Z_{F, T}=\int \exp \left(\mathscr{H}_{F, T}(\underline{x})\right) \rho^{T}(d \underline{x}) .
$$

In the same way we define $v_{F, T, z}, Z_{F, T, \underline{z}}, \hat{v}_{F, n}$, and $\hat{Z}_{F, n}$ by replacing $\mathscr{H}_{F, T}$ in (2.7) and (2.8) with $\mathscr{H}_{F, T}$ and $\hat{\mathscr{H}}_{F, n}$. We also consider the finite volume canonical Gibbs 
measures $v_{F, T}^{y}$ and $v_{F, T, z}^{y}$ which are obtained by conditioning the measures $v_{F, T}$ and $v_{F, T, \underline{z}}$ respectively, with respect to $m_{T}(\underline{x})=y$, where

$$
m_{T}(\underline{x})=\frac{1}{|T|} \sum_{a \in T} x_{a} .
$$

We define $\hat{v}_{F, n}^{y}$ in the same way by choosing $T=S_{n}^{d}$ in (2.9).

Note that a calculation like (2.15) below ensures the existence of a version of the conditioned measure $v_{F, T}^{y}$ which is continuous in $y$.

The family of the translation invariant (infinite volume) Gibbs measures and canonical Gibbs measures are defined by

$$
\begin{aligned}
\mathscr{G}_{F} & =\left\{\mu \in \mathscr{M}_{\tau} \mid \mu\left(\cdot \mid x_{a}=z_{a} \text { for } a \notin T\right)=v_{F, T, \underline{z}} \text { for all } \underline{z} \text { and finite } T \subset \mathbb{Z}^{d}\right\}, \\
\mathscr{G}_{F}^{c} & =\left\{\mu \in \mathscr{M}_{\tau} \mid \mu\left(\cdot \mid x_{a}=z_{a} \text { for } a \notin T \text { and } m_{T}(\cdot)=y\right)\right. \\
& \left.=v_{F, T, \underline{z}}^{y} \text { for all } \underline{z} \text { and finite } T \subset \mathbb{Z}^{d}\right\} .
\end{aligned}
$$

We denote the distribution of $R_{n, \underline{x}}$ with respect to $v_{F, T_{n}}$, by $P_{F, n}$ or simply $P_{n}$ when there is no danger of confusion. That is

$$
P_{n}(A)=v_{F, T_{n}}\left(R_{n, \underline{x}} \in A\right)
$$

for $A$ a Borel subset of $\mathscr{M}_{\tau}$. The probability measures $P_{F, n, \underline{z}}^{y}$ and $P_{n, \underline{z}}$ are defined in the same way. It is known [9] that the family $P_{n}$ has the large deviation property with the rate function $I_{F}$. In order to define $I_{F}(\mu)$ for $\mu \in \mathscr{M}_{\tau}$, we first define the pressure of an interaction $F$ by

$$
\Psi(F)=\lim _{n \rightarrow \infty} \frac{1}{\left|T_{n}\right|} \log Z_{F, T_{n}, \underline{z}} .
$$

Note that this limit is uniform in $\underline{z}$, and $\Psi(F)$ is independent of $\underline{z}$.

The specific entropy of a measure $\mu$ in $\mathscr{M}_{\tau}$ with respect to $\rho$ is defined as

$$
H_{\rho}(\mu)=\lim _{n \rightarrow \infty} \frac{1}{\left|T_{n}\right|} \int\left(\log \frac{d \mu^{T_{n}}}{d \rho^{T_{n}}}\right) d \mu^{T_{n}},
$$

where the measure $\mu^{T_{n}}$ is the projection of $\mu$ on the space $X_{T_{n}}$. The rate function $I_{F}$ is defined by

$$
I_{F}(\mu)=H_{\rho}(\mu)-\int F d \mu+\Psi(F)
$$

or

$$
I_{F}(\mu)=\lim _{n \rightarrow \infty} \frac{1}{\left|T_{n}\right|} \int f_{n} \log f_{n} d v_{F, T_{n}},
$$

where $f_{n}$ is the density of the measure $\mu^{T_{n}}$ with respect to the Gibbs measure $v_{F, T_{n}}$.

If $\alpha$ and $\beta$ are two probability measures such that

for some density $f \in L^{1}(d \beta)$, then

$$
d \alpha=f d \beta
$$

$$
\int g d \alpha \leqq \log \int e^{g} d \beta+\int \log f d \alpha
$$

for any $g \in L^{1}(d \alpha)$. We will refer to this inequality as the basic entropy estimate. 
The large deviation property of the sequence $R_{n, \underline{x}}$ with respect to the Gibbs measure $v_{F, T_{n}}$ also implies the large deviation property of the distributions of the sequence $m_{T_{n}}(\underline{x})$. For this we use the Contraction Principle.

Lemma 2.4. Let $\underline{z}^{n}$ be an arbitrary sequence in $X$ and $F \in C_{\mathrm{loc}}^{0}$. Then the family $\alpha_{n}(d y)=v_{F, T_{n} \geq_{n}}\left(m_{T_{n}} \in d y\right)$ has the large deviation property with the rate $\hat{I}_{F}(y)=$ $\inf I_{F}(\mu)$, where $\Phi(\mu)=\int x_{0} d \mu$ for those measures $\mu$ such that $\int\left|x_{0}\right| d \mu$ is finite.

Proof. Set $C_{l}=\left\{\mu \mid \int w\left(x_{0}\right) \mu(d \underline{x}) \leqq l\right\}$. Note that the set $C_{l}$ is closed in $X$ and $\Phi$ restricted to $C_{l}$ is continuous. Now we can apply Lemma 2.1. For this we need to check $P_{n}\left(X-C_{l}\right)$ is superexponentially small as $l \rightarrow \infty$. For notational convenience we denote $v_{F, T_{n}, \underline{z}}$ by $v_{n}$. Using the Tchebyshev inequality,

$$
P_{n}\left(X-C_{l}\right)=v_{n}\left\{\underline{x}: \frac{1}{\left|T_{n}\right|} \sum_{a \in T_{n}} w\left(x_{a}\right)>l\right\} \leqq e^{-l\left|T_{n}\right|} E^{v_{n}} \exp \left(\sum_{a \in T_{n}} w\left(x_{a}\right)\right) .
$$

On the other hand

$$
\begin{aligned}
& \underset{n \rightarrow \infty}{\limsup } \frac{1}{\left|T_{n}\right|} \log E^{v_{n}} \exp \left(\sum_{a \in T_{n}} w\left(x_{a}\right)\right) \\
& \quad=\limsup _{n \rightarrow \infty} \frac{1}{\left|T_{n}\right|} \log \int \exp \left(\sum_{a \in T_{n}}\left(w\left(x_{a}\right)-\phi\left(x_{a}\right)\right)\right) \exp \left(\sum_{a \in T_{n}} F\left(\tau_{a} \underline{x}\right)\right) d x-\Psi(F) \\
& \quad \leqq\|F\|_{\infty}+\log \int e^{w(x)-\phi(x)} d(x)-\Psi(F)<\infty .
\end{aligned}
$$

Thus

$$
\limsup _{l \rightarrow \infty} \limsup _{n \rightarrow \infty} \frac{1}{\left|T_{n}\right|} \log P_{n}\left(X-C_{l}\right)=-\infty,
$$

and this completes the proof.

Once we establish the large deviation property of the sequence $m_{T_{n}}(\underline{x})$ with respect to Gibbs measures, it is not hard to identify the rate function as the convex conjugate $h$ of the free energy $\varphi$ defined by

$$
\varphi(\lambda)=\varphi(F, \lambda)=\limsup _{n \rightarrow \infty} \frac{1}{\left|T_{n}\right|} \log \int \exp \left(\lambda \sum_{a \in T_{n}} x_{a}\right) v_{F, T_{n} \geq \underline{z}_{n}}(d \underline{x}) .
$$

\section{Lemma 2.5 .}

$$
\hat{I}_{F}(y):=h(y)=\sup _{y}(\lambda y-\varphi(\lambda)) .
$$

Proof. It is enough to show that

$$
\varphi(\lambda)=\sup _{y}\left(\lambda y-\hat{I}_{F}(y)\right)
$$

We only verify

$$
\varphi(\lambda) \leqq \sup _{y}\left(\lambda y-\hat{I}_{F}(y)\right)
$$


We omit the easy proof of

$$
\varphi(\lambda) \geqq \sup _{y}\left(\lambda y-\hat{I}_{F}(y)\right)
$$

which follows from the lower bound large deviation estimate.

Let $C_{l}$ and $v_{n}$ be as in Lemma 2.4. Since the function $\Phi$ (defined by $\Phi(\mu)=\int x_{0} d \mu$ ) restricted to the set $C_{l}$ is continuous, by Varadhan's theorem ([13]) we have

$$
\limsup _{n \rightarrow \infty} \frac{1}{\left|T_{n}\right|} \log \int_{K_{l}} \exp \left(\lambda \sum_{a \in T_{n}} x_{a}\right) d v_{n} \leqq \sup _{y}\left(\lambda y-\hat{I}_{F}(y)\right),
$$

where $K_{l}=\left\{\underline{x}\left|\sum_{a \in T_{n}} w\left(x_{a}\right) \leqq l\right| T_{n} \mid\right\}$.

Therefore we only need to show

$$
\limsup _{l \rightarrow \infty} \limsup _{n \rightarrow \infty} \frac{1}{\left|T_{n}\right|} \log \int_{K_{l}^{c}} \exp \left(\lambda \sum_{a \in T_{n}} x_{a}\right) v_{n}(d \underline{x})=-\infty
$$

or

$$
\limsup _{l \rightarrow \infty} \limsup _{n \rightarrow \infty} \frac{1}{\left|T_{n}\right|} \log \int_{K_{l}^{c}} \exp \left(\sum_{a \in T_{n}} w\left(x_{a}\right)\right) v_{n}(d \underline{x})=-\infty .
$$

But this is an easy consequence of the Tchebyshev inequality and (2.6).

Corollary 2.6. The family $\mu_{n}$ has the large deviation property with rate $h$.

Now we state the main result of this section.

Theorem 2.7. Let $\left\{\underline{z}_{n}\right\}$ be a sequence of the boundary conditions and $\left\{y_{n}\right\}$ be a sequence of real numbers such that $\lim _{n \rightarrow \infty} y_{n}=y$. Then the family $\left\{P_{F, T_{n}, \underline{z}_{n}}^{y_{n}}\right\}$ has the upper bound large deviation property with rate

$$
I_{F}^{y}(\mu)= \begin{cases}I_{F}(\mu)-h(y), & \text { if } \int x_{0} d \mu=y \\ \infty, & \text { otherwise }\end{cases}
$$

The proof of this Theorem is carried out in several steps.

Let $G \in C_{\text {loc }}^{1}$. Set

$$
\hat{G}_{n}(y)=\frac{1}{\left|T_{n}\right|} \log \int \exp \left(\mathscr{H}_{G, T_{n}}(\underline{x})\right) v_{F, T_{n} \underline{z}_{n}}^{y}(d \underline{x}) .
$$

Let $g_{n}(F, \underline{z} ; y)$ denote the density of $m_{T_{n}}$ with respect to $v_{F, T_{n}, \underline{z}}$. Then

Lemma 2.8 .

$$
\begin{aligned}
\hat{G}_{n}(y)= & \frac{1}{\left|T_{n}\right|} \log g_{n}\left(F+G, \underline{z}_{n} ; y\right)-\frac{1}{\left|T_{n}\right|} \log g_{n}\left(F, \underline{z}_{n} ; y\right) \\
& +\frac{1}{\left|T_{n}\right|} \log Z_{F+G, T_{n}, \underline{z}_{n}}-\frac{1}{\left|T_{n}\right|} \log Z_{F, T_{n} \underline{z}_{n}} .
\end{aligned}
$$


Proof. Let $J$ be a test function,

$$
\begin{aligned}
& \int J(y) g_{n}\left(F, \underline{z}_{n} ; y\right) \exp \left(\left|T_{n}\right| \hat{G}_{n}(y)\right) d y \\
& \quad=\int J\left(m_{T_{n}}(\underline{x})\right) \exp \left(\mathscr{H}_{G, T_{n}, \underline{z}_{n}}(\underline{x})+\mathscr{H}_{F, T_{n}, \underline{z}_{n}}(\underline{x})\right) \frac{1}{Z_{F}} d \rho^{T_{n}} ; \\
& \quad=\frac{Z_{F+G}}{Z_{F}} \int J\left(m_{T_{n}}\right) \exp \left(\mathscr{H}_{F+G, T_{n}, \underline{z}_{n}}\right) \frac{1}{Z_{F+G}} d \rho^{T_{n}} ; \\
& =\frac{Z_{F+G}}{Z_{F}} \int J(y) g_{n}\left(F+G, \underline{z}_{n} ; y\right) d y .
\end{aligned}
$$

Here to simplify the notations we have dropped the indices $\underline{z}$ and $T_{n}$ from $Z$.

The main ingredient to prove Theorem 2.7 is the following Lemma:

Lemma 2.9. The family $\widehat{G}_{n}$ is equicontinuous on every compact subset of $\mathbf{R}$.

Proof: Step 1. Because of Lemma 2.8, it is enough to show that the sequence

$$
\frac{1}{\left|T_{n}\right|} \frac{g_{n}^{\prime}(y)}{g_{n}(y)}
$$

is uniformly bounded over any compact subset of $\mathbf{R}$. Here, for ease in notation we have used $g_{n}$ for $g_{n}\left(G, \underline{z}_{n} ; y\right)$.

Let $J$ be a test function. Then we have

$$
\begin{aligned}
\frac{1}{\left|T_{n}\right|} \int J(y) g_{n}^{\prime}(y) d y & =-\frac{1}{\left|T_{n}\right|} \int J^{\prime}(y) g_{n}(y) d y \\
& =-\frac{1}{\left|T_{n}\right|} \int J^{\prime}\left(\frac{\sum_{a \in T_{n}} x_{a}}{\left|T_{n}\right|}\right) \exp \left(\mathscr{H}_{G, T_{n}}(\underline{x})-\sum_{a \in T_{n}} \phi\left(x_{a}\right)\right) \frac{1}{Z_{G, T_{n}, \underline{z}_{n}}} d \underline{x} \\
& =\int J\left(\frac{\sum_{a \in T_{n}} x_{a}}{\left|T_{n}\right|}\right)\left\{\frac{1}{\left|T_{n}\right|} \sum_{a \in T_{n}} \frac{\partial \mathscr{H}}{\partial x_{a}}(\underline{x})-\frac{1}{\left|T_{n}\right|} \sum_{a \in T_{n}} \phi^{\prime}\left(x_{a}\right)\right\} d v_{G, T_{n}, \underline{z}_{n}} \\
& =\int J(y) g_{n}(y)\left\{\int \frac{1}{\left|T_{n}\right|} \sum_{a \in T_{n}}\left(\frac{\partial \mathscr{H}_{n}}{\partial x_{a}}(\underline{x})-\phi^{\prime}\left(x_{a}\right)\right) d v_{G, T_{n}, \underline{z}_{n}}^{y}\right\} d y .
\end{aligned}
$$

Therefore

$$
\frac{1}{\left|T_{n}\right|} \frac{g_{n}^{\prime}(y)}{g_{n}(y)}=\int \frac{1}{\left|T_{n}\right|} \sum_{a \in T_{n}}\left(\frac{\partial \mathscr{H}}{\partial x_{a}}-\phi^{\prime}\left(x_{a}\right)\right) d v_{G, T_{n}, z_{n}}^{y} .
$$

So

$$
\frac{1}{\left|T_{n}\right|}\left|\frac{g_{n}^{\prime}(y)}{g_{n}(y)}\right| \leqq c_{0}+\int \frac{1}{\left|T_{n}\right|} \sum_{a \in T_{n}}\left|\phi^{\prime}\left(x_{a}\right)\right| d v_{G, T_{n}, \underline{z}_{n}}^{y},
$$

where $c_{0}$ is a constant depending only on $G$. 
Step 2. Let

$$
K_{n}(y)=\int \frac{1}{\left|T_{n}\right|} \sum_{a \in T_{n}}\left|\phi^{\prime}\left(x_{a}\right)\right| d v_{G, T_{n}, \underline{z}_{n}}^{y}(d \underline{x}) .
$$

It is easy to see that

$$
v_{G, T_{n}, \underline{z}_{n}}^{y}(d \underline{x})=\frac{1}{\tilde{Z}_{y}} \exp \left(\mathscr{H}_{G, T_{n}, \underline{z}_{n}}(\underline{x})\right) v_{n}^{y}(d \underline{x}) .
$$

Here $v_{n}^{y}(d \underline{x})$ is $v_{G, T_{n}, \underline{z}_{n}}^{y}(d \underline{x})$, where we set $G=0$, and $\tilde{Z}_{y}$ is the obvious normalizing constant. Now we use the basic entropy estimate to deduce

$$
\begin{aligned}
K_{n}(y)= & \int\left(\frac{1}{\left|T_{n}\right|} \sum_{a \in T_{n}}\left|\phi^{\prime}\left(x_{a}\right)\right|\right) \exp \left(\mathscr{H}_{G, T_{n}, \underline{z}_{n}}(\underline{x})\right) \frac{1}{\tilde{Z}_{y}} v_{n}^{y}(d \underline{x}) \\
\leqq & \frac{1}{\left|T_{n}\right|} \log \int \exp \left(\sum_{a \in T_{n}}\left|\phi^{\prime}\left(x_{a}\right)\right|\right) v_{n}^{y}(d \underline{x}) \\
& +\frac{1}{\left|T_{n}\right|} \int \log \frac{\exp \left(\mathscr{H}_{G, T_{n}, \underline{z}_{n}}(\underline{x})\right)}{\widetilde{Z}_{y}} v_{G, T_{n}, \underline{z}_{n}}^{y}(d \underline{x}) \\
= & \mathscr{I}_{1}+\mathscr{I}_{2} .
\end{aligned}
$$

On the other hand

$$
\left|\mathscr{H}_{G, T_{n}, \underline{z}_{n}}\right| \leqq c_{1}\left|T_{n}\right|,
$$

where $c_{1}$ is a constant depending only on $G$. Therefore

$$
\tilde{Z}_{y}=\int \exp \left(\mathscr{H}_{G, T_{n}, \underline{z}_{n}}\right) v_{n}^{y}(d \underline{x}) \geqq e^{-c_{1}\left|T_{n}\right|}
$$

So

$$
\mathscr{I}_{2} \leqq 2 c_{1}
$$

Final Step. In order to finish the proof, all we need is to show that the term $\mathscr{I}_{1}$ is uniformly bounded in $y$ for $y$ in a compact subset of $\mathbf{R}$. Let $J$ be a test function as before, and let $k_{n}(y)$ be the density of $m_{T_{n}}(\underline{x})$ with respect to $\rho^{T_{n}}(d \underline{x})=$ $\exp \left(-\sum_{a \in T_{n}} \phi\left(x_{a}\right)\right) d \underline{x}$. Then

$$
\begin{aligned}
& \int k_{n}(y) J(y)\left(\int \exp \left(\sum_{a \in T_{n}}\left|\phi^{\prime}\left(x_{a}\right)\right|\right) v_{n}^{y}(d \underline{x})\right) d y \\
& \quad=\int J\left(m_{T_{n}}(\underline{x})\right) \exp \left(\sum_{a \in T_{n}}\left(\left|\phi^{\prime}\left(x_{a}\right)\right|-\phi\left(x_{a}\right)\right)\right) \prod_{a \in T_{n}} d x_{a} \\
& =M^{\left|T_{n}\right|} \int J\left(m_{T_{n}}(\underline{x})\right) \prod_{a \in T_{n}} \frac{e^{\left|\phi^{\prime}\left(x_{a}\right)\right|-\phi\left(x_{a}\right)}}{M} d x_{a} \\
& =M^{\left|T_{n}\right|} \int J(y) \tilde{k}_{n}(y) d y,
\end{aligned}
$$


where $\tilde{k}_{n}(y)$ is the density of $m_{T_{n}}(\underline{x})$ with respect to the product measure

$$
\prod_{a \in T_{n}} \frac{e^{\left|\phi^{\prime}\left(x_{a}\right)\right|-\phi\left(x_{a}\right)}}{M} d x_{a}
$$

and

$$
M=\int e^{\left|\phi^{\prime}(x)\right|-\phi(x)} d x
$$

Therefore

$$
\frac{1}{\left|T_{n}\right|} \log \int \exp \left(\sum_{a \in T_{n}}\left|\phi^{\prime}\left(x_{a}\right)\right|\right) v_{n}^{y}(d \underline{x})=\frac{1}{\left|T_{n}\right|} \log \tilde{k}_{n}(y)-\frac{1}{\left|T_{n}\right|} \log k_{n}(y)+M .
$$

Note that we can write

$$
\prod_{a \in T_{n}} \frac{e^{\left|\phi^{\prime}\left(x_{a}\right)\right|-\phi\left(x_{a}\right)}}{M} d x_{a}=\prod_{a \in T_{n}} e^{-\tilde{\phi}\left(x_{a}\right)} d x_{a},
$$

where

$$
\tilde{\phi}(x)=-\left|\phi^{\prime}(x)\right|+\phi(x)+\log M .
$$

So, by (2.18) the term $\mathscr{I}_{1}$ is bounded if we can show $\left(1 /\left|T_{n}\right|\right) \log k_{n}(y)$ is bounded and $\left(1 /\left|T_{n}\right|\right) \log \tilde{k}_{n}(y)$ is bounded above. We will show these in Lemmas 2.10 and 2.11 .

Lemma 2.10. $\left(1 /\left|T_{n}\right|\right) \log k_{n}(y)$ is bounded over any compact subset of $\mathbf{R}$.

Proof. This immediately follows from Theorem 3.4 in [4].

Lemma 2.11. There exists a positive constant $c_{2}$ such that

$$
\tilde{k}_{n}(y) \leqq c_{2}\left|T_{n}\right|
$$

for all $n \geqq 1$.

Proof. Let $f=e^{-\tilde{\phi}}$. First we show $f \in L^{2}(\mathbf{R})$. Because of (2.5), it suffices to check that $e^{-\phi}$ is uniformly bounded. This follows from the integrability of the derivative of $e^{-\phi}$,

$$
\int\left|\phi^{\prime}\right| e^{-\phi} \leqq \log \int e^{\left|\phi^{\prime}\right|-\phi}<\infty .
$$

Since $f \in L^{2}(\mathbf{R})$, we have $f * f \in L^{\infty}$. This implies that the $n$-fold convolution of $f$ is bounded by $\|f * f\|_{\infty}$ for $n \geqq 2$. Therefore the density of $\sum_{a \in T_{n}} x_{a}$ is also uniformly bounded for all $n \geqq 1$. Thus $\tilde{k}_{n}(y)$ is bounded by $\left|T_{n}\right|\|f * f\|_{\infty}$.

An easy consequence of Lemma 2.9 and Corollary 2.6 is the following theorem which is a large deviation result for the densities $g_{n}(F, \underline{z} ; y)$.

Theorem 2.12. Let $F \in C_{\mathrm{loc}}^{1}$. Then

$$
\lim _{n \rightarrow \infty} \frac{1}{\left|T_{n}\right|} \log g_{n}(F, \underline{z} ; y)=-h(y)
$$

uniformly in boundary conditions $\underline{z}$ and uniformly in $y$ in compact intervals of $\mathbf{R}$. 
Proof. It suffices to show

$$
\lim _{n \rightarrow \infty} \frac{1}{\left|T_{n}\right|} \log g_{n}\left(F, z_{n} ; y\right)=-h(y)
$$

uniformly on compact $y$-intervals, where $\left\{\underline{z}_{n}\right\}$ is any sequence of boundary conditions.

By Lemma 2.10 the sequence $\left\{\left(1 /\left|T_{n}\right|\right) \log g_{n}\left(F, \underline{z}_{n} ; y\right)\right\}$ is equicontinuous over compact intervals. Now we show that this sequence is bounded over any compact $y$-intervals. By (2.15) we have

$$
\begin{aligned}
& \frac{1}{\left|T_{n}\right|} \log \int \exp \left(\mathscr{H}_{F, T_{n}, \underline{z}_{n}}(\underline{x})\right) v_{n}^{y}(d \underline{x}) \\
& \quad=\frac{1}{\left|T_{n}\right|} \log g_{n}\left(F, \underline{z}_{n} ; y\right)-\frac{1}{\left|T_{n}\right|} \log k_{n}(y)+\log Z_{F, T_{n}, \underline{z}_{n}},
\end{aligned}
$$

where $v_{n}^{y}$ and $k_{n}$ are as in the proof of Lemma 2.9. Since the right-hand side of the above identity is uniformly bounded in $y$, and since the sequence $\left(1 /\left|T_{n}\right|\right) \log k_{n}(y)$ is uniformly bounded over compact $y$-intervals (Lemma 2.10), therefore the sequence $\left\{\left(1 /\left|T_{n}\right|\right) \log g_{n}\left(F, \underline{z}_{n} ; y\right)\right\}$ is also uniformly bounded over compact $y$ intervals.

If a subsequence of $\left\{\left(1 /\left|T_{n}\right|\right) \log g_{n}\left(F, \underline{z}_{n} ; y\right)\right\}$ converges to a function $g$, then by Corollary $2.6, g$ can only be $-h(y)$ and this completes the proof.

Now we check that $I_{F}^{y}$ is a rate function.

Lemma 2.13. The set $\left\{\mu: I_{F}^{y}(\mu) \leqq \ell\right\}$ is compact for all $\ell$.

Proof. Let $I_{F}^{y}(\mu)<\infty$. Then, by the basic entropy inequality

$$
\begin{aligned}
\int w\left(x_{0}\right) \mu(d \underline{x})= & \frac{1}{\left|T_{n}\right|} \int\left(\sum_{a \in T_{n}} w\left(x_{a}\right)\right) \mu(d \underline{x}) \\
= & \frac{1}{\left|T_{n}\right|} \int\left(\sum_{a \in T_{n}} w\left(x_{a}\right)\right) \mu^{T_{n}}(d \underline{x}) \\
\leqq & \frac{1}{\left|T_{n}\right|} \log \int \exp \left(\sum_{a \in T_{n}} w\left(x_{a}\right)\right) \rho^{T_{n}}(d \underline{x}) \\
& +\frac{1}{\left|T_{n}\right|} \int \log \frac{d \mu^{T_{n}}}{d \rho^{T_{n}}}(\underline{x}) \mu^{T_{n}}(d \underline{x}) .
\end{aligned}
$$

Therefore, after passing to limit as $n \rightarrow \infty$,

$$
\begin{aligned}
\int w\left(x_{0}\right) \mu(d \underline{x}) & \leqq \log \int e^{w(x)-\phi(x)} d x+H_{\rho}(\mu) \\
& =\log \int e^{w(x)-\phi(x)} d x+I_{F}(\mu)+\int F d \mu-\psi(F) \\
& \leqq c_{0}+I_{F}(\mu),
\end{aligned}
$$

where $c_{0}$ is a constant depending on $F$.

This implies that $\int w\left(x_{0}\right) \mu(d x)$ is bounded if $I_{F}(\mu)$ is bounded. Note that $\Phi(\mu)=\int x_{0} d \mu$ is continuous if it is restricted to the set $\left\{\mu: \int w\left(x_{0}\right) \mu(d x) \leqq \ell_{0}\right\}$ for 
any $\ell_{0}$,

$$
\begin{aligned}
\left\{\mu: I_{F}^{y}(\mu) \leqq \ell\right\}= & \left\{\mu: \int x_{0} d \mu=y \text { and } I_{F}(\mu) \leqq \ell+h(y)\right\} \\
= & \left\{\mu: \int x_{0} d \mu=y \text { and } \int w\left(x_{0}\right) \leqq c_{0}+\ell+h(y)\right\} \\
& \cap\left\{\mu: I_{F}(\mu) \leqq \ell+h(y)\right\} .
\end{aligned}
$$

Thus the set $\left\{\mu: I_{F}^{y}(\mu) \leqq \ell\right\}$ is compact, because $I_{F}$ is a rate function.

Proof of Theorem 2.7. Step 1. We use Lemma 2.3, in order to show the inequality (2.2) (i.e. the upper bound estimate) for the family $\left\{P_{F, T_{n}, z_{n}}^{y_{n}}\right\}$. For this, we need to consider a suitable closed base for the topology of the weak convergence. Let $\mathscr{A}_{0}$ be the collection of the closed sets of the form

$$
\left\{\mu \in \mathscr{M}_{\tau}(X): \int G_{1} d \mu \in I_{1}, \int G_{2} d \mu \in I_{2} \cdots \int G_{k} d \mu \in I_{k}\right\},
$$

where $I_{1}, \ldots, I_{k}$ are fixed closed subsets of $\mathbf{R}$ and $G_{1}, \ldots, G_{k}$ are in $C_{\text {loc }}^{1}$. We also use the compact notation $\underline{G}=\left(G_{1}, \ldots, G_{k}\right)$ in the sequel.

Note that if $A_{1}, \ldots, A_{k}$ are in $\mathscr{A}_{0}$ then $A_{1} \cap \cdots \cap A_{m}$ is also in $\mathscr{A}_{0}$. Let $\mathscr{A}$ be the collection of all finite unions of the elements of $\mathscr{A}_{0}$. That is,

$$
\mathscr{A}=\left\{B_{1} \cup \cdots \cup B_{k} \mid \text { for some } B_{1}, B_{2}, \ldots, B_{k} \text { in } \mathscr{A}_{0}\right\} \text {. }
$$

It is easy to check that $\mathscr{A}$ is a topological closed base for $\mathscr{M}_{\tau}(X)$. Since the space $\mathscr{M}_{\tau}(X)$ is separable, then for any closed subset $C$ of $\mathscr{M}_{t}(X)$, there exists a decreasing sequence $\left\{A_{n}\right\}$ in $\mathscr{A}$ such that $C=\cap_{n} A_{n}$.

Step 2. Consider the continuous function $\Phi: \mathscr{M}_{\tau}(x) \rightarrow \mathbf{R}^{k}$ defined as

$$
\Phi(\mu)=\int \underline{G} d \mu .
$$

For ease in notation, we set $v_{n}^{y_{n}}=v_{F, T_{n}, \underline{z}_{n}}^{y_{n}}$. Let $\underline{\theta}=\left(\theta_{1}, \ldots, \theta_{k}\right) \in \mathbf{R}^{k}$. We define

$$
\begin{aligned}
\xi(\underline{\theta} ; y) & =\xi\left(\theta_{1}, \ldots, \theta_{k} ; y\right)=\lim _{n \rightarrow \infty} \frac{1}{\left|T_{n}\right|} \log \int \exp \left(\left|T_{n}\right|(\underline{\theta} \cdot \Phi(\mu)) P_{F, T_{n}, \underline{z}_{n}}^{y_{n}}(d \mu)\right. \\
& =\lim _{n \rightarrow \infty} \frac{1}{\left|T_{n}\right|} \int \exp \left(\mathscr{H}_{\underline{\theta} \cdot \underline{G}, T_{n}}\right) d v_{n}^{y_{n}} .
\end{aligned}
$$

The last equality needs some explanation. From the definition of $P_{F, T_{n}, \underline{z}_{n}}^{y_{n}}$ we obtain the last term with $\mathscr{H}_{\underline{\theta} \cdot \underline{G}, T_{n}, \underline{z}_{n}}$. But, after passing to limit, the contribution of the boundary condition disappears. Now we use (2.15) to conclude

$$
\begin{aligned}
\xi(\underline{\theta} ; y)= & \lim _{n \rightarrow \infty} \frac{1}{\left|T_{n}\right|} \log g_{n}\left(\underline{\theta} \cdot \underline{G}+F, \underline{z}_{n} ; y\right) \\
& -\lim _{n \rightarrow \infty} \frac{1}{\left|T_{n}\right|} \log g_{n}\left(F, \underline{z}_{n} ; y\right) \\
& +\Psi(\underline{\theta} \cdot \underline{G}+F)-\Psi(F) .
\end{aligned}
$$

Let $\varphi(\cdot, \lambda)$ be defined as in $(2.13)$ and let $h(\cdot ; y)$ be the convex conjugate of $\varphi(\cdot ; \lambda)$ in the variable $\lambda$. By Theorem 2.12,

$$
\xi(\underline{\theta} ; y)=-h(\underline{\theta} \cdot \underline{G}+F ; y)+h(F ; y)+\Psi(\underline{\theta} \cdot \underline{G}+F)-\Psi(F) .
$$


Step 3. By Lemma 2.2, the family

$$
\nu_{n}^{y_{n}}\left(\int \underline{G} d \mu \in d \underline{t}\right)
$$

has the upper bound large deviation property with rate

$$
I(\underline{t} ; y)
$$

which is the convex conjugate of $\xi(\underline{\theta} ; y)$ in the variable $\underline{\theta}$. More precisely

$$
\begin{aligned}
I(\underline{t} ; y) & =\sup _{\underline{\theta}}(\underline{\theta} \cdot \underline{t}-\xi(\underline{\theta} ; y)) \\
& =\sup _{\underline{\theta}}\left(\underline{\theta} \cdot \underline{t}+\sup _{\lambda}(\lambda y-\varphi(\underline{\theta} \cdot \underline{G}+F ; \lambda))-\Psi(\underline{\theta} \cdot \underline{G}+F)+\Psi(F)\right)-h(y) \\
& =\sup _{\underline{\theta}, \lambda}(\underline{\theta} \cdot \underline{t}+\lambda y-\varphi(\underline{\theta} \cdot \underline{G}+F ; \lambda)-\Psi(F+\underline{\theta} \cdot \underline{G})+\Psi(F))-h(y) .
\end{aligned}
$$

Step 4. Let $\hat{\Phi}(\mu)=\left(\int \underline{G} d \mu, \int x_{0} d \mu\right)$. We have shown the Contraction Principle for $\Phi$ in Lemma 2.4 (when $\underline{G}=0$ ). However, with the same argument, we have the same result for $\hat{\Phi}$ for any $\underline{G}$ in $C_{\text {loc }}^{1}$. We can also identify the rate function as in Lemma 2.5 . Thus the family $\left\{P_{F, T_{n} z_{n}} \circ \widehat{\Phi}^{-1}\right\}$ has the large deviation property with the rate function

$$
\begin{aligned}
\hat{I}(\underline{t} ; y) & =\inf \left\{I_{F}(\mu): \int \underline{G} d \mu=\underline{t} \text { and } \int x_{0} d \mu=y\right\} \\
& =\text { convex conjugate of } \hat{\varphi}(\underline{\theta} ; \lambda) \text { in } \underline{\theta} \text { and } \lambda,
\end{aligned}
$$

where

$$
\begin{aligned}
\hat{\varphi}(\underline{\theta} ; \lambda) & =\lim _{n \rightarrow \infty} \frac{1}{\left|T_{n}\right|} \log \int \exp \left(\lambda \sum_{a \in T_{n}} x_{a}+\mathscr{H}_{\underline{\theta} \cdot \underline{G}}(\underline{x})\right) v_{F, T_{n}, \underline{z}_{n}}(d \underline{x}) \\
& =\lim _{n \rightarrow \infty} \frac{1}{\left|T_{n}\right|} \log \int \exp \left(\lambda \sum_{a \in T_{n}} x_{a}\right) v_{F+\underline{\theta} \cdot \underline{G}, T_{n}, \underline{z}_{n}}(d \underline{x})-\Psi(F)+\Psi(\underline{\theta} \cdot \underline{G}+F) \\
& =\varphi(F+\underline{\theta} \cdot \underline{G} ; \lambda)-\Psi(F)+\Psi(\underline{\theta} \cdot \underline{G}+F) .
\end{aligned}
$$

Thus

$$
I(\underline{t} ; y)=\hat{I}(\underline{t} ; y)-h(y) .
$$

Final Step. Let $A=\left\{\mu: \int G_{1} d \mu \in I_{1}, \ldots, \int G_{k} d \mu \in I_{k}\right\}$ as in the beginning of the proof. Then

$$
\begin{aligned}
& \limsup _{n \rightarrow \infty} \frac{1}{\left|T_{n}\right|} \log P_{F, T_{n} \underline{z}_{n}}^{y_{n}}(A) \leqq-\inf \left\{I(\underline{t} ; y): t_{1} \in I_{1}, \ldots, t_{k} \in I_{k}\right\} \text { by step 3, } \\
& =-\inf \left\{\hat{I}(\underline{t} ; y): t_{1} \in I_{1}, \ldots, t_{k} \in I_{k}\right\}+h(y) \\
& =-\inf _{\substack{\mu \in A \\
\int x_{0} d \mu=y}}\left(I_{F}(\mu)-h(y)\right) \\
& =-\inf _{\mu \in A} I_{F}^{y}(\mu) \text {. }
\end{aligned}
$$

Since any set in $\mathscr{A}$ is a finite union of the sets in $\mathscr{A}_{0}$, we also have the above inequality for $A \in \mathscr{A}$. 

by $\mathscr{G}_{\lambda}$.

Let $F \in C_{\text {loc }}^{1}$. For each $\lambda$, set $F_{\lambda}(\underline{x})=F(\underline{x})+\lambda x_{0}$. For simplicity we denote $\mathscr{G}_{F_{\lambda}}$

Theorem 2.14. Suppose that $G \in C_{\mathrm{loc}}^{1}$ or $G(\underline{x})=G_{1}(\underline{x})+\phi^{\prime}\left(x_{0}\right)$ for some $G_{1} \in C_{\mathrm{loc}}^{0}$. Let $\underline{z}_{n}$ be a sequence in $X$, let $y_{n}$ be a sequence of real numbers such that $\lim y_{n}=y$, and let $F \in C_{\text {loc }}^{1}$. Then the family

$$
\alpha_{n}(d t)=v_{F, T_{n}, \underline{n}_{n}}^{y_{n}}\left\{\frac{1}{\left|T_{n}\right|} \sum_{a \in T_{n}} G\left(\tau_{\alpha} \underline{x}\right) \in d t\right\}
$$

has the upper bound large deviation property with rate

$$
J_{G}(t)=\inf \left\{I_{F}^{y}(\mu): \int G d \mu=t\right\} .
$$

Moreover $J_{G}(t)=0$ if and only if $t=\int G d \mu$ for some $\mu \in \mathscr{G}_{h^{\prime}(y)}$ with $\int x_{0} d \mu=y$.

Proof. If $G \in C_{\mathrm{loc}}^{0}$, then the large deviation property is an immediate consequence of the Contraction Principle and Theorem 2.7. If $G(\underline{x})=G_{1}(\underline{x})+\phi^{\prime}\left(x_{0}\right)$ for some $G_{1} \in C_{\mathrm{loc}}^{0}$, we may apply Lemma 2.1. For this we need to show that

$$
\limsup _{\ell \rightarrow \infty} \limsup \frac{1}{\left|T_{n}\right|} \log v_{F, T_{n} \geq_{n}}^{y_{n}}\left(\frac{1}{\left|T_{n}\right|} \sum_{a \in T_{n}} w\left(\phi^{\prime}\left(x_{a}\right)\right) \geqq \ell\right)=-\infty .
$$

Because the function $\Phi(\mu)=\int G d \mu$, restricted to the set $\left\{\mu: \int w\left(\phi^{\prime}\left(x_{0}\right)\right) \mu(d \underline{x}) \leqq \ell\right\}$ is continuous.

Formula (2.19) follows from

$$
\limsup _{n \rightarrow \infty} \frac{1}{\left|T_{n}\right|} \log \int \exp \left(\sum_{a \in T_{n}} w\left(\phi^{\prime}\left(x_{a}\right)\right)\right) d v_{F, T_{n} \geq_{n}}^{y_{n}} \leqq+\infty
$$

and the Tchebychev inequality. For this, we can essentially repeat our argument in the final step of the proof of Lemma 2.9 (see (2.18)).

If $J_{G}(t)=0$, then there exists a measure $\mu$ in $\mathscr{M}_{\tau}(X)$ such that

$$
\int x_{0} d \mu=y ; \quad \int G d \mu=t ; \quad \text { and } \quad I_{F}(\mu)=h(y) .
$$

On the other hand by (2.11) we have

$$
\begin{aligned}
I_{F_{\lambda}}(\mu) & =H_{\rho}(\mu)-\int F d \mu-\lambda \int x_{0} d \mu+\Psi\left(F_{\lambda}\right) \\
& =I_{F}(\mu)-\left\{\lambda y-\left(\Psi\left(F_{\lambda}\right)-\Psi(F)\right)\right\} .
\end{aligned}
$$

From the definition of the function $h$ as the convex conjugate of $\varphi$, we have

$$
h(y)=\sup _{\lambda}(\lambda y-\varphi(F ; \lambda))=\sup _{\lambda}\left(\lambda y-\Psi\left(F_{\lambda}\right)+\Psi(F)\right),
$$

and the supremum is attained at $\lambda=h^{\prime}(y)$. Therefore

$$
h(y)=h^{\prime}(y) y-\Psi\left(F_{h^{\prime}(y)}\right)+\Psi(F) .
$$

From this, (2.21) and (2.22), we obtain

$$
I_{F_{\lambda}}(\mu)=0 \quad \text { if } \quad \lambda=h^{\prime}(y) .
$$

Finally, it follows from the Variational Principle (see Preston [10], p. 115) that $\mu \in \mathscr{G}_{h^{\prime}(y)}$. 
Remark 2.15. We have assumed that $h$ is differentiable. Since $h$ is convex, this is equivalent to strict convexity of $\varphi(\lambda)$ or $\Psi\left(F_{\lambda}\right)$ in $\lambda$. This also follows from the Variational Principle. See Preston [10], p. 158 for more details.

Corollary 2.16. If $G_{0}(\underline{x})=\phi^{\prime}\left(x_{0}\right)-\left(\partial \mathscr{H}_{n} / \partial x_{0}\right)(\underline{x})$, where $\mathscr{H}_{n}=\mathscr{H}_{F, T_{n}}$, then $J_{G_{0}}(t)=0$ if and only if $t=h^{\prime}(y)$.

Proof. Let $\mu \in \mathscr{G}_{\lambda}$ for some $\lambda \in \mathbf{R}$. Then

$$
\begin{aligned}
\int G_{0} d \mu & =-\int\left(\frac{\partial \mathscr{H}_{n}}{\partial x_{0}}(\underline{x})-\phi^{\prime}\left(x_{0}\right)\right) \mu(d \underline{x}) \\
& =-\int \frac{1}{\left|T_{n}\right|} \sum_{a \in T_{n}}\left(\frac{\partial \mathscr{H}_{n}}{\partial x_{a}}(\underline{x})-\phi^{\prime}\left(x_{a}\right)\right) \mu(d \underline{x}) \\
& =-\int \mu(d \underline{z}) \int \frac{1}{\left|T_{n}\right|} \sum_{a \in T_{n}}\left(\frac{\partial \mathscr{H}_{n}}{\partial x_{a}}(\underline{x})-\phi^{\prime}\left(x_{a}\right)\right) d v_{F_{\lambda}, T_{n}, \underline{z}} \\
& =\lambda+r_{n},
\end{aligned}
$$

where $\lim _{n \rightarrow \infty} r_{n}=0$. This is because

$$
d v_{F_{\lambda}, T_{n}, \underline{z}}=\frac{1}{Z_{F_{\lambda}, T_{n}, \underline{z}_{n}}} \exp \left(\tilde{\mathscr{H}}_{n}(\underline{x})-\sum_{a \in T_{n}} \phi^{\prime}\left(x_{a}\right)+\lambda \sum_{a \in T_{n}} x_{a}\right),
$$

where $\tilde{\mathscr{H}}_{n}=\mathscr{H}_{F, T_{n}, \underline{z}}$. The last equality in (2.23) follows from integration by parts. The error $r_{n}$ comes from the boundary condition $\underline{z}$ when we replace $\partial \tilde{\mathscr{H}}_{n} / \partial x_{a}$ with $\partial \mathscr{H}_{n} / \partial x_{a}$.

Now let $\lambda=h^{\prime}(y)$ and pass to limit $n \rightarrow \infty$ in (2.23).

Theorem 2.17. Let $K$ be a bounded subset of $\mathbf{R}$, and let $G_{0}$ be as in Corollary 2.16 . Then

$$
\lim _{n \rightarrow \infty} \sup _{\substack{\underline{z} \in X \\ y \in K}} \int\left|\frac{1}{\left|T_{n}\right|} \sum_{a \in T_{n}} G_{0}\left(\tau_{a} \underline{x}\right)-h^{\prime}(y)\right| d v_{F, T_{n}, \underline{\underline{z}}}^{y}=0 .
$$

Proof. It follows from Theorem 2.14 and its corollary that

$$
\lim _{n \rightarrow \infty} v_{F, T_{n}, \underline{z}_{n}}^{y_{n}}\left(\left|\frac{1}{\left|T_{n}\right|} \sum_{a \in T_{n}} G_{0}\left(\tau_{a} \underline{x}\right)-h^{\prime}(y)\right|>\delta\right)=0,
$$

where $y_{n} \rightarrow y, \underline{z}_{n}$ is an arbitrary sequence in $X$, and $\delta>0$. Therefore, for (2.24) we only need to check the uniform integrability of the sequence

$$
\frac{1}{\left|T_{n}\right|} \sum_{a \in T_{n}} G_{0}\left(\tau_{a} \underline{x}\right)
$$

with respect to the measures $v_{F, T_{n}, \underline{z}_{n}}^{y_{n}}$. For this, it suffices to show

$$
\sup _{n} \int w\left(\frac{1}{\left|T_{n}\right|} \sum_{a \in T_{n}} \phi^{\prime}\left(x_{a}\right)\right) d v_{F, T_{n}, \underline{z}_{n}}^{y_{n}}<\infty .
$$


Since $w$ is convex, it is enough to show

$$
\sup _{n} \int \frac{1}{\left|T_{n}\right|} \sum_{a \in T_{n}} w\left(\phi^{\prime}\left(x_{a}\right)\right) d v_{F, T_{n}, \underline{z}_{n}}^{y_{n}}<\infty .
$$

But this is an immediate consequence of (2.20) and Jensen's inequality.

Theorem 2.18. Let $F \in C_{\text {loc }}^{1}$. Then

$$
\frac{1}{\left|T_{n}\right|} \frac{g_{n}^{\prime}(y)}{g_{n}(y)} \rightarrow-h^{\prime}(y)
$$

uniformly in $\underline{z} \in X$ and $y$ in a compact subset of $\mathbf{R}$, where

$$
g_{n}(y)=g_{n}(F, \underline{z} ; y) .
$$

Proof. This follows from Theorem 2.17 and (2.16).

The function $G_{0}$ is of very special form. That is why we have an ergodic theorem like Theorem 2.16 even when phase transition occurs (i.e. $\mathscr{G}_{\lambda}$ for some $\lambda$ is not singleton). In general we do not expect to have such a result for any $G \in C_{\mathrm{loc}}^{0}$. If we however assume that the space of Gibbs measures has a linear structure, we can extend Theorem 2.16 to any $G \in C_{\mathrm{loc}}^{1}$. Note that this assumption does not exclude phase transition.

Definition 2.19. We say that $\mathscr{G}_{\lambda}$ has linear structure if $\mu_{1}, \mu_{2} \in \mathscr{G}_{\lambda}$ and $\int x_{0} d \mu_{1}=$ $\int x_{0} d \mu_{2}$, then $\mu_{1}=\mu_{2}$.

If $\mathscr{G}_{\lambda}$ has linear structure for all $\lambda$, then, for any $G \in C_{\text {loc }}^{0}$, we can define

$$
A_{G}(y)=\int G d \mu,
$$

where $\mu$ is the unique measure in $\mathscr{G}_{h^{\prime}(y)}$ such that $\int x_{0} d \mu=y$.

Theorem 2.20. Under the above assumption,

$$
\lim _{n \rightarrow \infty} \sup _{\substack{z \in X \\ y \in K}} \int\left|\frac{1}{\left|T_{n}\right|} \sum_{a \in T_{n}} G\left(\tau_{a} \underline{x}\right)-A_{G}(y)\right| d v_{F, T_{n}, \underline{z}}^{y}=0,
$$

where $K$ is any bounded subset of $\mathbf{R}$.

Proof. This follows from Theorem 2.14 and the proof to Theorem 2.17.

In Sect. 5 we will need the following estimate.

Lemma 2.21. There exists a constant $c$ such that

$$
\left|h^{\prime}(y)\right| \leqq c+h(y) .
$$

Proof. Let $G_{0}$ be as in Corollary 2.16, and let $\mu \in \mathscr{G}_{h^{\prime}(y)}$ be such that $\int x_{0} d \mu=y$. Let $\lambda=h^{\prime}(y)$. Using the basic entropy estimate we have

$$
\begin{aligned}
\int \frac{1}{\left|T_{n}\right|} \sum_{a \in T_{n}}\left|\phi^{\prime}\left(x_{a}\right)\right| d v_{F_{\lambda}, T_{n}, \underline{z}} \leqq & \frac{1}{\left|T_{n}\right|} \log \int \exp \left(\sum_{a \in T_{n}}\left|\phi^{\prime}\left(x_{a}\right)\right|\right) d \rho^{T_{n}} \\
& +\frac{1}{\left|T_{n}\right|} \int\left(\mathscr{H}_{F_{\lambda}, T_{n}, \underline{z}}-\log Z_{F_{\lambda}, T_{n}, \underline{\underline{z}}}\right) d v_{F_{\lambda}, T_{n}, \underline{\underline{z}}} .
\end{aligned}
$$


By integrating both sides in $\underline{z}$ with respect to $\mu$ and passing to the limit, we obtain

$$
\begin{aligned}
\limsup _{n \rightarrow \infty} \int\left(\frac{1}{\left|T_{n}\right|} \sum_{a \in T_{n}}\left|\phi^{\prime}\left(x_{a}\right)\right|\right) \mu(d \underline{x}) & \leqq \log \int e^{\left|\phi^{\prime}(x)\right|-\phi(x)} d x+\int F d \mu+\lambda \int x_{0} d \mu-\Psi\left(F_{\lambda}\right) \\
& =c_{1}+\lambda \int x_{0} d \mu-\Psi\left(F_{\lambda}\right)+\Psi(F) \\
& \leqq c_{1}+h(y),
\end{aligned}
$$

where $c_{1}$ is a suitable constant and $\lambda=h^{\prime}(y)$. On the other hand, by our calculation in Corollary 2.16 we have

$$
\left|h^{\prime}(y)\right| \leqq c_{2}+\limsup _{n \rightarrow \infty} \int\left(\frac{1}{\left|T_{n}\right|} \sum_{a \in T_{n}}\left|\phi^{\prime}\left(x_{a}\right)\right|\right) d \mu
$$

for some constant $c_{2}$. This will give us (2.25) with $c=c_{1}+c_{2}$.

\section{A Local Ergodic Theorem}

Initially we start from a distribution having density $f_{N}^{0}$ with respect to the Gibbs measure $v_{N}$ with periodic boundary condition (see (1.12)) The initial density $f_{N}^{0}$ satisfies the entropy condition

$$
\frac{1}{N^{d}} \int f_{N}^{0} \log f_{N}^{0} d v_{N} \leqq C .
$$

Then the evolution governed by the generator $\mathscr{L}_{N}$ (see (1.10)) gives us the density $f_{N}^{t}$ which satisfies the forward equation

$$
\frac{\partial f_{N}^{t}}{\partial t}=\mathscr{L}_{N} f_{N}^{t}
$$

Then for large $N$ the measure $f_{N}^{t} d v_{N}$ locally looks somewhat like a Gibbs measure with the interaction $F$ and a chemical potential $\lambda=h^{\prime}(y)$, where $y$ corresponds to the macroscopic charge density, coming from the averages $m_{T}$. To make this precise, we first state Theorem 3.1 which is some kind of ergodic theorem.

Let $Q_{N}$ be the law of the diffusion generated by $\mathscr{L}_{N}$ on the space $C\left([0, \infty), X_{N}\right)$ where $X_{N}=\mathbf{R}^{S_{N}^{d}}$.

Theorem 3.1. Let $G_{0}$ be as in Corollary 2.16, and let $T_{\ell}(a)=a+T_{\ell}$. Then

$$
\begin{gathered}
\lim _{k \rightarrow \infty} \limsup _{\varepsilon \rightarrow 0} \limsup _{\ell \rightarrow \infty} \limsup _{N \rightarrow \infty} \frac{1}{N^{d}} \sum_{a \in S_{N}^{d}} E^{Q_{N}} \int_{0}^{t} \mid \frac{1}{\left|T_{\ell}(a)\right|} \sum_{u \in T_{\ell}(a)} G_{0}\left(\tau_{u} \underline{x}(s)\right) \\
-\psi_{k}{ }^{\circ} h^{\prime}\left(m_{T_{N \varepsilon}(a)}(\underline{x}(s))\right) \mid d s=0,
\end{gathered}
$$

where

$$
\psi_{k}(x)= \begin{cases}x & |x| \leqq k \\ k & x>k \\ -k & x<-k\end{cases}
$$


If we set

$$
\hat{f}_{N}^{t}(\underline{x})=\frac{1}{t} \int_{0}^{t} f_{N}^{s}(\underline{x}) d s
$$

and

$$
\eta_{N}(\underline{x})=\frac{1}{N^{d}} \sum_{a \in S_{N}^{d}} \hat{f}_{N}^{t}\left(\tau_{a} \underline{x}\right)
$$

for $\underline{x} \in \mathbf{R}^{S_{N}^{d}}$, then (3.1) can be written as

$\lim _{k \rightarrow \infty} \limsup _{\varepsilon \rightarrow 0} \limsup \limsup _{\ell \rightarrow \infty} E^{\eta_{N} d v_{N}} \mid \frac{1}{\left|T_{\ell}\right|} \sum_{u \in T_{\ell}} G_{0}\left(\tau_{u} \underline{x}\right)-\psi_{k}{ }^{\circ} h^{\prime}\left(m_{T_{N \varepsilon}}(\underline{x}) \mid=0\right.$.

The proof of (3.3) is carried out in several steps. Let $P^{N}$ denote $\eta_{N} d v_{N}$.

Theorem 3.2. For any $\delta>0$,

$$
\lim _{t \rightarrow \infty} \limsup _{N \rightarrow \infty} P^{N}\left\{\underline{x}:\left|\frac{1}{\left|T_{\lambda}\right|} \sum_{u \in T_{\ell}} G_{0}\left(\tau_{u} \underline{x}\right)-h^{\prime}\left(m_{T_{\ell}}(\underline{x})\right)\right|>\delta\right\}=0 .
$$

Before proving Theorem 3.2, we study the limit points of the sequence $\eta_{N} d v_{N}$. For this we follow [4]. First we define

$$
\mathscr{J}^{N}(\mu)=\frac{1}{N^{2}} \sup _{f>0}\left[\int \frac{-\mathscr{L}_{N} f}{f} d \mu\right]
$$

for $\mu$ a measure on the space $\mathbf{R}^{S_{N}^{d}}$. Then

Lemma 3.3. (a)

$$
\frac{1}{N^{d}} \int \eta_{N} \log \eta_{N} d v_{N} \leqq C
$$

(b)

$$
\frac{1}{N^{d}} \mathscr{J}^{N}\left(P^{N}\right) \leqq \frac{C}{4 N^{2} t}
$$

We omit the proof, which follows the proof of Theorem 2.4 in [4].

Let $\operatorname{Proj}_{\ell}$ denote the projection on the space $\mathbf{R}^{T_{\ell}}$. Let $\mathscr{T}$ be the set of measures $\mu \in \mathscr{M}_{\tau}(X)$ such that for each $\ell, \operatorname{Proj}_{\ell}(\mu)$ is a weak limit point of the sequence $\operatorname{Proj}_{\ell}\left(P^{N}\right)$.

Lemma 3.4. (a)

$$
\sup _{\mu \in \mathscr{T}} \sup _{\ell} E^{\mu} \frac{1}{\left|T_{\ell}\right|} \sum_{a \in T_{\ell}} w\left(x_{a}\right)<\infty
$$

(b)

$$
\sup _{\mu \in \mathscr{T}} \sup _{\ell} E^{\mu} \frac{1}{\left|T_{\ell}\right|} \sum_{a \in T_{\ell}} w\left(\phi^{\prime}\left(x_{a}\right)\right)<\infty
$$

(c)

$$
\mathscr{T} \subseteq \mathscr{G}_{F}^{c}
$$


Proof. (a) is an easy consequence of (3.4) and the basic entropy estimate. (b) also follows from this estimate

$$
\begin{aligned}
E^{P^{N}} \frac{1}{\left|T_{\ell}\right|} \sum_{a \in T_{\ell}} w\left(\phi^{\prime}\left(x_{a}\right)\right) & =E^{P^{N}} \frac{1}{N^{d}} \sum_{a \in S_{N}^{d}} w\left(\phi^{\prime}\left(x_{a}\right)\right) \\
& \leqq \frac{1}{N^{d}} \log \int \exp \left(\sum w\left(\phi^{\prime}\left(x_{a}\right)\right) d v_{N}+\frac{1}{N^{d}} \int \eta_{N} \log \eta_{N} d v_{N} .\right.
\end{aligned}
$$

This is uniformly bounded in $N$ because of (3.4) and (2.6).

(c) Consider the following diffusion generator

$$
\mathscr{L}_{a, b}=\frac{1}{2}\left(\frac{\partial}{\partial x_{a}}-\frac{\partial}{\partial x_{b}}\right)^{2}-\frac{1}{2}\left(\phi^{\prime}\left(x_{a}\right)-\phi^{\prime}\left(x_{b}\right)-\frac{\partial \mathscr{H}_{N}}{\partial x_{a}}+\frac{\partial \mathscr{H}_{N}}{\partial x_{b}}\right)\left(\frac{\partial}{\partial x_{a}}-\frac{\partial}{\partial x_{b}}\right) .
$$

For finite $T \cong \mathbb{Z}^{d}$, we define

$$
\mathscr{L}_{T}=\sum \mathscr{L}_{a, b},
$$

where the sum is over bonds $a, b$ (adjacent sites) in $T$. Corresponding to $\mathscr{L}_{T}$, we associate the form

$$
\mathscr{J}_{\mathrm{T}}(\mu)=\sup _{f>0}\left[\int \frac{-\mathscr{L}_{T} f}{f} d \mu\right]
$$

where $\mu \in \mathscr{M}\left(\mathbf{R}^{S}\right)$ for some $S$ with $T+\Lambda \cong S(\Lambda$ is the range of $F)$. When $\mu \in \mathscr{M}\left(\mathbf{R}^{S}\right)$ then the supremum in (3.6) is over $f \in C^{\infty}\left(\mathbf{R}^{S}, \mathbf{R}\right)$. Note that

$$
\begin{aligned}
& \mathscr{J}^{N}(\mu)=\mathscr{J}_{S_{N}^{d}}(\mu)=\sum \mathscr{J}_{a, b}(\mu), \\
& \mathscr{J}^{N}(\mu)=\sum \mathscr{E}_{a, b}^{N}\left(\sqrt{\eta_{N}}\right),
\end{aligned}
$$

where

$$
\mathscr{E}_{a, b}^{N}(f)=\frac{1}{2} \int\left(\frac{\partial f}{\partial x_{a}}-\frac{\partial f}{\partial x_{b}}\right)^{2} d v_{N} .
$$

From these considerations and Lemma 3.3, we obtain

$$
\mathscr{E}_{a, b}^{N}\left(\sqrt{\eta_{N}}\right) \leqq \frac{C}{4 N^{2} t d}
$$

for $a, b$ adjacent sites (this is because $\eta_{N}$ is translation invariant in the space $\mathbf{R}^{S_{N}^{d}}$ ). This implies

$$
\mathscr{J}_{a, b}\left(P^{N}\right) \leqq \frac{C}{4 N^{2} t d}
$$

(for the relation between $\mathscr{J}_{a, b}$ and $\mathscr{E}_{a, b}^{N}$ in general see Stroock [12], Theorem 7.44).

Suppose that $a+\Lambda, b+\Lambda \cong T_{\ell}$. Then, in (3.6) with $T=\{a, b\}$, if we restrict the supremum to functions $f \in C^{\infty}\left(\mathbf{R}^{T_{t}}, \mathbf{R}\right)$ and $f>0$, we obtain

$$
\mathscr{J}_{a, b}\left(\operatorname{Proj}_{\ell}\left(P^{N}\right)\right) \leqq \mathscr{J}_{a, b}\left(P^{N}\right) \text {. }
$$


Thus

$$
\mathscr{J}_{a, b}\left(\operatorname{Proj}_{\ell}\left(P^{N}\right)\right) \leqq \frac{C}{4 N^{2} t d}
$$

Let $\mu \in \mathscr{T}$. Then by lower semicontinuity of $\mathscr{J}_{a, b}$, we have

$$
\mathscr{J}_{a, b}\left(\operatorname{Proj}_{\ell} \mu\right)=0 \text {. }
$$

Thus

$$
\mathscr{J}_{T}\left(\operatorname{Proj}_{\ell} \mu\right)=0
$$

for any finite set $T \subseteq \mathbb{Z}^{d}$. In Lemma 3.5, we will show that (3.9) implies (c) and this completes the proof.

Lemma 3.5. The following statements are equivalent:

(a) $\mathscr{J}_{T}\left(\operatorname{Proj}_{\ell} \mu\right)=0$ for all large $\ell$ such that $T+\Lambda \subseteq T_{\ell}$, and all finite sets $T \leqq \mathbb{Z}^{d}$;

(b) $\int \mathscr{L}_{T} f d \mu=0$, for all $f \in C_{\mathrm{loc}}^{\infty}$ and all finite sets $T \subseteq \mathbb{Z}^{d}$;

(c) $\mu \in \mathscr{G}_{F}^{c}$.

Proof. $(\mathrm{a} \Leftrightarrow \mathrm{b})$ Consider the inequalities

$$
\int \frac{\mathscr{L}_{T} f}{f} d \mu \geqq 0
$$

for $f \in C_{\mathrm{loc}}^{\infty}, f>0$ and $T \subseteq \mathbb{Z}^{d}$ finite. By setting $f=e^{t u}$ with $u \in C_{\mathrm{loc}}^{\infty}$ and $t \in \mathbf{R}$, and then varying $t$, it is easy to see that (3.10) is equivalent to

$$
\int \mathscr{L}_{T} u d \mu=0
$$

for all $u \in C_{\text {loc }}^{\infty}$ and $T \subseteq \mathbb{Z}^{d}$ finite.

$(\mathrm{b} \Leftrightarrow \mathrm{c})$ Let $\mathscr{F}_{T}$ be the $\sigma$-field generated by the variables $x_{a}$ with $a \notin T$, and $m_{T}(\underline{x})$. Then, if $f, g \in C_{\text {loc }}^{\infty}$ and $g$ is $\mathscr{F}_{T}$-measurable, we have

$$
\mathscr{L}_{T}(f g)=g \mathscr{L}_{T} f
$$

Given $\mu \in \mathscr{M}_{\tau}(X)$, let $\mu_{T, \underline{z}}^{y}$ denote the conditional probability distribution of $\mu$ given $\mathscr{F}_{T}$. More precisely

$$
\mu_{T, \underline{z}}^{y}(d \underline{x})=\mu\left(d \underline{x} \mid x_{a}=z_{a} \quad \text { for } a \notin T, \quad \text { and } \quad m_{T}(\underline{x})=y\right),
$$

where $y=m_{T}(\underline{z})$. From (3.12), we conclude that (3.11) is equivalent to

$$
\int \mathscr{L}_{T} f d \mu_{T, \underline{z}}^{y}=0
$$

for all $f \in C_{\text {loc }}^{\infty}$, and almost all $\underline{z}$ with respect to $\mu$ (here, we have used the fact that we only need to verify (3.13) for a suitable countable family of functions $f$ ).

It is not hard to see that $\mathscr{L}_{T}$ acting on the space of functions defined for the variables $x_{a}$, for all $a \in T$ and $m_{T}(\underline{x})=y$ is elliptic with the unique invariant measure $v_{T, \underline{z}}^{y}$. Thus (3.13) is equivalent to

$$
v_{T, \underline{z}}^{y}=\mu_{T, \underline{z}}^{y} \quad \text { a.a. } \quad \underline{z},
$$

and this completes the proof. 
Proof of Theorem 3.2. On account of Theorem 2.16 and Lemma 3.4 (c), we only need to check

$$
\limsup _{p \rightarrow \infty} \lim \sup _{\ell \rightarrow \infty} \sup _{\mu \in \mathscr{T}} \int_{\left|m_{T_{\ell}}(\underline{z})\right| \geqq p} \mu(d \underline{z})=0 .
$$

But this is an immediate consequence of Lemma 3.4 (a), because $w$ in this lemma satisfies (2.6).

What we have shown so far is an ergodic theorem for the averages of $G_{0}$ over the microscopically large blocks of $\mathbb{Z}^{d}$. In Theorem 3.1, however, we need to show that these averages are close to the chemical potential of a macroscopic charge density. The gap between Theorems 3.1 and 3.2 is filled by the following result:

Theorem 3.6. For any $\delta>0$,

$$
\lim _{\varepsilon \rightarrow 0} \limsup _{i \rightarrow \infty} \limsup _{N \rightarrow \infty} P^{N}\left\{\underline{x}: \mid h^{\prime}\left(m_{T_{f}}(\underline{x})-h^{\prime}\left(m_{T_{N \varepsilon}}(\underline{x}) \mid>\delta\right\}=0 .\right.\right.
$$
lemma.

For this, we first link a block to a macroscopically close block by the following

Lemma 3.7. For any $\delta>0$

$$
\lim _{\varepsilon \rightarrow 0} \limsup _{i \rightarrow \infty} \limsup _{N \rightarrow \infty} \sup _{v} P^{N}\left\{\underline{x}: \mid h^{\prime}\left(m_{T_{t}}(\underline{x})-h^{\prime}\left(m_{T_{\lambda}(v)}(\underline{x})\right) \mid>\delta\right\}=0,\right.
$$

where the supremum is over sites $v$ such that $T_{\ell}(v) \subseteq T_{N \varepsilon}$, and

( $\Lambda$ is the range of $F$ ).

Proof. Let $d \beta_{N, \ell, v}$ be the projection of $\eta_{N} d v_{N}$ on the space $\mathbf{R}^{T_{f}+\Lambda} \times \mathbf{R}^{T_{(}(v)+\Lambda}$, where $v$ may depend on $N, \ell$ and $\varepsilon$. After a change of variable we view this measure as a measure on the space $\mathbf{R}^{T_{C}+\Lambda} \times \mathbf{R}^{T_{(}\left(\mathrm{v}_{0}\right)+\Lambda}$, where $v_{0}$ is now a fixed site such that

$$
\left(T_{\ell}\left(v_{0}\right)+\Lambda\right) \cap\left(T_{\ell}+\Lambda\right)=\varnothing .
$$

We abuse the notation and denote this measure by $\beta_{N, \ell, v_{0}}$.

Let $\beta$, be any limit point of $\beta_{N, \ell, v_{0}}$ as $N$ goes to infinity. Then by (3.9) and its consequence (3.11) we have

$$
\int \mathscr{L}_{T_{\ell}} f d \beta_{\ell}=\int \mathscr{L}_{T_{\ell}\left(v_{0}\right)} f d \beta_{\ell}=0
$$

for all $f \in C^{\infty}\left(\mathbf{R}^{T_{t}+\Lambda} \times \mathbf{R}^{T_{f}\left(v_{0}\right)+\Lambda}, \mathbf{R}\right)$. The proof of Lemma 3.5 implies that

$$
d \beta_{\ell}=\int v_{F, T_{\ell, \underline{z}}}^{y_{1}} \nu_{F, T_{\ell}\left(v_{0}\right), \underline{z}}^{y_{2}} \gamma\left(d y_{1}, d y_{2}, d \underline{z}\right)
$$

for some probability measure $\gamma$. We also consider $\mathscr{L}_{0, v}$ and $\mathscr{E}_{0, v}^{N}$, where $\Lambda \subseteq T_{\ell}$, $T_{\ell}(v) \subseteq T_{N \varepsilon}$. Then, the proof of Lemma 2.3 in [4] implies that $\mathscr{E}_{0, v}^{N}\left(\eta_{N}\right) \leqq C \varepsilon^{2} d / 4 t$. Therefore

$$
\begin{gathered}
\mathscr{J}_{0, v}\left(P^{N}\right) \leqq \frac{C \varepsilon^{2} d}{4 t}, \\
\mathscr{J}_{0, v}\left(\beta_{\mathrm{N}, l, v}\right) \leqq \frac{C \varepsilon^{2} d}{4 t} .
\end{gathered}
$$


Then by lower semicontinuity of $\mathscr{J}_{0, v}$ (or $\left.\mathscr{J}_{0, v_{0}}\right)$,

$$
\mathscr{J}_{0, v_{0}}\left(\beta_{\ell}\right) \leqq \frac{C \varepsilon^{2} d}{4 t} .
$$

Let $\hat{\beta}_{\ell}\left(d y_{1}, d y_{2}\right)$ be the distribution of $m_{T_{t}}$ and $m_{T_{\ell}\left(v_{0}\right)}$ with respect to $\beta_{\ell}$. Our Theorem 2.18 and (3.15) allow us to follow the proof of Theorem 4.3 in [4] and conclude that any $\hat{\beta}$, a limit point of $\hat{\beta}_{\ell}$ as $\ell$ goes to infinity, satisfies

$$
\int_{\mathbf{R}^{2}}\left|h^{\prime}\left(y_{1}\right)-h^{\prime}\left(y_{2}\right)\right|^{2} \hat{\beta}\left(d y_{1}, d y_{2}\right) \leqq \frac{2 C \varepsilon^{2} d}{t} .
$$

Now let $\varepsilon \rightarrow 0$ in (3.16). This will prove (3.14).

Proof of Theorem 3.6. Let

$$
B_{N, \ell, \varepsilon}=\left\{v \in S_{N}^{d}: T_{\ell}(v) \cong T_{N \varepsilon} \text { and }\left(T_{\ell}+\Lambda\right) \cap\left(T_{\ell}(v)+\Lambda\right)=\varnothing\right\} .
$$

Let $M_{N, \ell}(v)$ denote the indicator function of the set

$$
\left\{\underline{x}:\left(m_{T_{\ell}}(\underline{x}), m_{T_{C(v)}}(\underline{x})\right) \in A_{\delta / 2}\right\},
$$

where

$$
A_{\delta}=\left\{(x, y) \in \mathbf{R}^{2}:\left|h^{\prime}(x)-h^{\prime}(y)\right| \geqq \delta\right\} .
$$

We simplify the notation by dropping indices $N, \ell$ and $\varepsilon$, from $B$ and $M(v)$. We will also denote lim lim sup lim sup by 'Lims'. After these conventions, Lemma 3.7 says $\varepsilon \rightarrow 0 \quad \ell \rightarrow \infty \quad N \rightarrow \infty$

$$
\text { Lims } \sup _{v \in B} E^{P^{N}} M(v)=0 .
$$

Since

$$
E^{P^{N}} \frac{1}{|T|} \sum_{a \in T} w\left(x_{a}\right) \leqq C
$$

for any $T \subseteq S_{N}^{d}$, we obtain

$$
\operatorname{Lims} \sup _{v \in B} E^{P^{N}} M(v)\left|m_{T_{(}(v)}\right|=0 .
$$

Therefore

$$
\begin{gathered}
\operatorname{Lims} E^{P^{N}} \frac{1}{|B|} \sum_{v \in B} M(v)=0, \\
\operatorname{Lims} E^{P^{N}} \frac{1}{|B|} \sum_{v \in B} M(v)\left|m_{T_{N(v)}}\right|=0 .
\end{gathered}
$$

From (3.19) we conclude

$$
\operatorname{Lims} E^{P N}\left|m_{T_{N \varepsilon}}-\frac{1}{|B|} \sum_{v \in B}(1-M(v)) m_{T_{C}(v)}\right|=0 .
$$


Denoting $\sum_{v \in B} M(v)$ by $\hat{M}$, we can write

$$
\operatorname{Lims} P^{N}\left(\left|1-\frac{|B|}{|B|-\hat{M}}\right|>\delta^{\prime}\right)=0
$$

for any $\delta^{\prime}>0$ (because of (3.18)). This and the uniform integrability property (3.17) imply

$$
\operatorname{Lims} P^{N}\left(\left|\frac{1}{|B|} \sum_{v \in B}(1-M(v)) m_{T_{\ell}(v)}-\frac{1}{|B|-\hat{M}} \sum_{v \in B}(1-M(v)) m_{T_{\ell}(v)}\right|>\delta^{\prime}\right)=0
$$

for $\delta^{\prime}>0$. Let $W=\frac{1}{|B|-\hat{M}} \sum_{v \in B}(1-M(v)) m_{T_{\ell}(v)}$. Then by (3.20),

$$
\operatorname{Lims} P^{N}\left(\left|m_{T_{N r}}-W\right|>\delta^{\prime}\right)=0 .
$$

Since $W$ is a convex combination of random variables $m_{T_{f}(v)}$ such that

$$
\left(m_{T_{\ell}}, m_{T_{\ell}(v)}\right) \notin A_{\delta / 2},
$$

we have

$$
\left(m_{T_{\ell}}, W\right) \notin A_{\delta / 2} .
$$

This is simply because the set $\left\{y:(x, y) \notin A_{\delta / 2}\right\}$ is an interval for fixed $x \in \mathbf{R}$. Finally (3.21) guarantees

$$
\operatorname{Lims} P^{N}\left(\left(m_{T_{\ell}}, m_{T_{N \varepsilon}}\right) \in A_{\delta}\right) \leqq \operatorname{Lims} P^{N}\left(\left(m_{T_{N \varepsilon}}, W\right) \in A_{\delta / 2}\right)=0,
$$

and this is precisely the content of the theorem.

Proof of Theorem 3.1. It follows from what we have just proved and Theorem 3.2

$$
\underset{\varepsilon \rightarrow 0}{\limsup } \limsup _{\ell \rightarrow \infty} \limsup _{N \rightarrow \infty} P^{N}\left(\left|\psi_{k}\left(\frac{1}{\left|T_{\ell}\right|} \sum_{u \in T_{\ell}} G_{0}\left(t_{u} \underline{x}\right)\right)-\psi_{k} \circ h^{\prime}\left(m_{T_{N \varepsilon}}(\underline{x})\right)\right|>\delta\right)=0 .
$$

Since $\psi_{k}$ is bounded, we can replace the convergence in probability with the convergence in expectation. Now all we need to complete the proof is the uniform integrability of the sequence

$$
\left\{\frac{1}{\left|T_{,}\right|} \sum_{u \in T_{\ell}} G_{0}\left(\tau_{u} \underline{x}\right)\right\}
$$

But this is just the role of Lemma 3.4 (b).

If we assume that $\mathscr{G}_{\lambda}$ has linear structure for all $\lambda$ (see Definition 2.19), then we can improve Theorem 3.1 by replacing $G_{0}$ with any $G \in C_{\text {loc }}^{0}$. Essentially, this is because $A_{G}(y)$ is continuous in $y$ and $A_{G}(y)$ is affine on the interval $\left\{y: h^{\prime}(y)=h^{\prime}(x)\right\}$ for each fixed $x$. These two properties of $A_{G}$ will be shown in Lemma 3.9.

Theorem 3.8. Let $G \in C_{\mathrm{loc}}^{0}$. Then

$$
\lim _{\varepsilon \rightarrow 0} \limsup _{N \rightarrow \infty} E^{\eta_{N} d v_{N}} \mid \frac{1}{\left|T_{N \varepsilon}\right|} \sum_{a \in T_{N \varepsilon}} G\left(\tau_{a}(\underline{x})-A_{G}\left(m_{T_{N \varepsilon}}\right) \mid=0 .\right.
$$


Proof. If we go through the proof of Theorem 3.1, we will see that we only need to verify

$$
\lim _{\varepsilon \rightarrow 0} \limsup \limsup _{\ell \rightarrow \infty} P^{N}\left(\left|\frac{1}{\left|T_{N \varepsilon}\right|} \sum_{v \in T_{N \varepsilon}} A_{G}\left(m_{T_{\ell}(v)}\right)-A_{G}\left(m_{T_{N \varepsilon}}\right)\right|>\delta\right)=0
$$

for any $\delta>0$.

We continue to use our compact notations like $B$ and Lims as in the proof of Theorem 3.6. We can write the content of this theorems as

$$
\text { Lims } \sup _{v \in B} P^{N}\left(\left|h^{\prime}\left(m_{T_{N \varepsilon}}\right)-h^{\prime}\left(m_{T_{(}(v)}\right)\right|>\delta\right)=0
$$

for any $\delta>0$. Let $K$ denote the (may be degenerate) interval $\left\{y: h^{\prime}(y)=h^{\prime}\left(m_{T_{N \varepsilon}}\right)\right\}$ and let $C_{v}$ be the closest point in $K$ to $m_{T_{\ell}(v)}$. Since $A_{G}$ is affine on $K$, we have

$$
A_{G}\left(\frac{1}{|B|} \sum_{v \in B} C_{v}\right)=\frac{1}{|B|} \sum_{v \in B} A_{G}\left(C_{v}\right) \text {. }
$$

It is not hard to see that (3.23) implies

$$
\begin{gathered}
\operatorname{Lims} P^{N}\left(\left|\frac{1}{|B|} \sum_{v \in B} C_{v}-m_{T_{N \varepsilon}}\right|>\delta\right)=0, \\
\operatorname{Lims} P^{N}\left(\left|\frac{1}{|B|} \sum_{v \in B} A\left(C_{v}\right)-\frac{1}{|B|} \sum_{v \in B} A\left(m_{T_{,}(v)}\right)\right|>\delta\right)=0
\end{gathered}
$$

for any $\delta>0$. We omit the proof of (3.25) and (3.26) which is very much like the proof of Theorem 3.6. Now (3.22) follows from (3.24), (3.25) and (3.26).

Lemma 3.9. (a) $A_{G}$ is a continuous function, and

(b) $A_{G}$ is affine on the interval $\left\{y: h^{\prime}(y)=h^{\prime}(x)\right\}$.

Proof. (a) Using the basic entropy estimate, it is not hard to see

$$
\sup _{\substack{\mu \in \mathscr{G}_{\lambda} \\ \lambda \in J}} f\left(x_{0}\right) d \mu<\infty,
$$

where $J$ is a bounded subset of $\mathbf{R}$. This implies that $\Phi(\mu)=\int x_{0} d \mu$ is a continuous function on the space $\bigcup_{\lambda \in J} \mathscr{G}_{\lambda}$. Now let $y_{n} \rightarrow y$ and $y_{n}=\int x_{0} d \mu_{n}$ for some $\mu_{n} \in \bigcup_{\lambda} \mathscr{G}_{\lambda}$. Then if $\mu$ is any weak limit point of the sequence $\mu_{n}$, we have

Thus

$$
y=\int x_{0} d \mu \text { and } \mu \in \bigcup_{\lambda} \mathscr{G}_{\lambda} .
$$

$$
\lim _{n \rightarrow \infty} A_{G}\left(y_{n}\right)=\lim _{n \rightarrow \infty} \int G d \mu_{n}=\int G d \mu=A_{G}(y) .
$$

(b) This follows from linearity of $\int G d \mu$ in $\mu$.

\section{Large Deviations for Empirical Measures}

In Sect. 5 we will use Theorem 3.1 to derive the hydrodynamic equation. This requires to replace back $\psi_{k}$ with $h^{\prime}$. This is the object of Theorem 4.1. 
Let $f_{N}$ be the density relative to $d v_{N}$ of a random variable on $\mathbf{R}^{S_{N}^{d}}$ such that

$$
\sup _{N} \frac{1}{N^{d}} \int f_{N} \log f_{N} d v_{N} \leqq C .
$$

Let $Q_{N}$ be the law of the empirical measure

$$
\mu=\frac{1}{N^{d}} \sum_{a \in S_{N}^{d}} x_{a} \delta_{a / N}
$$

where $x_{a}$ are distributed according to $f_{N} d v_{N}$.

Theorem 4.1. For any limit $Q$ of $Q_{N}$, we have

$$
Q\{\mu: \mu \ll d \theta\}=1
$$

and

$$
E^{Q}\left[\int h\left(\frac{d \mu}{d \theta}\right)\right] \leqq C .
$$

Here, $\mathrm{d} \theta$ denotes the probability Haar measure on the d-dimensional torus.

The proof of this follows Lemma 6.3 in [4] which uses the large deviation property of the empirical measures. When the interaction $F$ is not present (i.e. $v_{N}$ is a product measure), the large deviation property is shown in [13]. In Theorem 4.2 we show the upper bound large deviation property for empirical measures with respect to $v_{N}$. This is all we need to carry out the proof of Theorem 4.1.

Theorem 4.2. The family $Q_{N}$ has the upper bound large deviation property with the rate function

$$
J(\mu)= \begin{cases}\int h\left(\frac{d \mu}{d \theta}\right) d \theta & \text { if } \quad \mu \ll d \theta \\ +\infty & \text { otherwise }\end{cases}
$$

We recall the function $\varphi$ defined by

$$
\varphi(\lambda)=\lim _{n \rightarrow \infty} \frac{1}{\left|T_{n}\right|} \log \int \exp \left(\lambda \sum_{a \in T_{n}} x_{a}\right) d v_{N} .
$$

We first prove

Theorem 4.3. Let $f$ be a continuous function on the d-dimensional torus. Then

$$
\lim _{N \rightarrow \infty} \frac{1}{N^{d}} \log E^{v_{N}} \exp \left(\sum_{a \in S_{N}^{d}} f(a / N)\right)=\int \varphi(f(\theta)) d \theta .
$$

Proof. Let $\zeta(f)$ denote the left-hand side of (4.1). The idea is dividing $S_{N}^{d}$ into small pieces on which of each $f(a / N)$ is almost a constant. Then we can use $\varphi$ to calculate $\zeta(f)$.

Let $k$ be a fixed positive integer and let $\mathscr{C}_{k}^{N}$ be a collection of cubes of length $k$, all separated by corridors of width $\ell$, where $\ell$ is the length of $\Lambda$ ( $\Lambda$ is the range of $F)$. Let $\Gamma$ denote the union of the cubes in $\mathscr{C}_{k}^{N}$ and let $M_{k}^{N}$ be the total number of 
them. We may construct $\mathscr{C}_{k}^{N}$ such that

$$
\lim _{N \rightarrow \infty} \frac{k^{d} M_{k}^{N}}{N^{d}}=1
$$

Since $f$ and $F$ are bounded and since $\int e^{c|x|} \rho(d x)<\infty$ for all $c \in \mathbf{R}\left(\rho(d x)=e^{-\phi(x)} d x\right)$, we may drop all terms with $x_{a}, a \in S_{N}^{d}-\Gamma$ in (4.1), and obtain

$$
\zeta(f)=\lim _{N \rightarrow \infty} \frac{1}{N^{d}} \log \int \prod_{T \in \mathscr{G}_{R}^{N}}\left[\exp \left(\sum_{a \in T} f(a / N) x_{a}+\mathscr{H}_{F, T}(\underline{x})\right)\right] \rho^{T}(d \underline{x})-\psi(F) .
$$

Let $\omega(\delta)=\sup |f(t+h)-f(t)|$, where the supremum is over $t$ and over $|h|<\delta$. Let $b(T)$ denote the center of $T$. In (4.3) we like to replace $f(a / N)$ with $f(b(T) / N)$. Let $1<p<\infty$ and $1 / p+1 / q=1$. Then by the Schwartz inequality

$$
\int \exp \left[\sum_{a \in T}\left(f\left(\frac{a}{N}\right) x_{a}+\mathscr{H}_{F, T}(\underline{x})\right)\right] d \rho^{T} \leqq \mathscr{A}(N, T, p) \mathscr{B}(N, T, p),
$$

where

$$
\begin{aligned}
& \mathscr{A}(N, T, p)=\left[\int \exp \left(f\left(\frac{b(T)}{N}\right) \sum_{a \in T} x_{a}+p \mathscr{H}_{F, T}(\underline{x})\right) d \rho^{T}\right]^{1 / p} \\
& \mathscr{B}(N, T, p)=\left[\int\left(q \omega\left(\sqrt{d} \frac{k}{N}\right) \sum_{a \in T}\left|x_{a}\right|\right)\right]^{1 / q}
\end{aligned}
$$

Furthermore $\mathscr{A}(N, T, p) \leqq \mathscr{A}^{\prime}(N, T, p) \exp \left(|T|(p-1) / p\|F\|_{\infty}\right)$, where

$$
\mathscr{A}^{\prime}(N, T, p)=\left[\int \exp \left(p f\left(\frac{b(T)}{N}\right) \sum_{a \in T} x_{a}+\mathscr{H}_{F, T}(\underline{x})\right) d \rho^{T}\right]^{1 / p}
$$

and

$$
\underset{N \rightarrow \infty}{\limsup } \frac{1}{N^{d}} \log \prod_{T \in \mathscr{E}_{k}^{N}} \mathscr{B}(N, T, p)=\limsup _{N \rightarrow \infty} \frac{M_{k}^{N} k^{d}}{N^{d} q} \log \int e^{q \omega \sqrt{k / N})|x|} \rho(d x)=0
$$

Let $\varphi_{T}(\lambda)=\frac{1}{|T|} \log E^{v_{F}, T} \exp \left(\lambda \sum_{a \in T} x_{a}\right)$. Note that $\varphi_{T}(\lambda)$ only depends on $k$ the length of $T$. From (4.4) and (4.5) we conclude

$$
\zeta(f) \leqq \limsup \limsup _{p \rightarrow 1} \limsup _{N \rightarrow \infty} \frac{1}{N^{d}} \sum_{T \in \mathscr{C}_{k}^{N}} \log \mathscr{A}^{\prime}(N, T, p)-\Psi(F) .
$$

On the other hand

$$
\begin{aligned}
\frac{1}{|T|} \log \mathscr{A}^{\prime}(N, T, p) & =\frac{1}{p|T|} \log \int \exp \left(p f\left(\frac{b(T)}{N}\right) \sum_{a \in T} x_{a}\right) d v_{F, T}+\frac{1}{|T|} \log Z_{F, T} \\
& =\frac{1}{p} \varphi_{T}\left(p f\left(\frac{b(T)}{N}\right)\right)+\frac{1}{|T|} \log Z_{F, T} .
\end{aligned}
$$


Thus

$$
\begin{aligned}
\zeta(f) & \leqq \limsup _{p \rightarrow 1} \limsup _{k \rightarrow \infty} \limsup _{N \rightarrow \infty}\left\{\frac{1}{p} \frac{1}{N^{d}} \sum_{T \in \mathscr{C}_{k}^{N}} \varphi_{T}\left(p f\left(\frac{b(T)}{N}\right)\right)+\frac{1}{p} \frac{M_{k}^{N}}{N^{d}} \log Z_{F, T}\right\}-\psi(F) \\
& =\limsup _{p \rightarrow 1} \limsup _{k \rightarrow \infty} \frac{1}{p} \int \varphi_{T}(p f(\theta)) d \theta \\
& =\int \varphi(f(\theta)) d \theta .
\end{aligned}
$$

We can prove $\zeta(f) \geqq \int \varphi(f(\theta)) d \theta$ in the same way. First we apply the Schwarz inequality to write

$$
\begin{aligned}
& \int \exp \left(\sum_{a \in T} f\left(\frac{b(T)}{N}\right) x_{a}+\mathscr{H}_{F, T}(\underline{x})\right) d \rho^{T} \\
& \leqq\left[\int \exp \left(p \sum_{a \in T} f\left(\frac{a}{N}\right) x_{a}+p \mathscr{H}_{F, T}(\underline{x})\right) d \rho^{T}\right]^{1 / p} \mathscr{B}(N, T, p) .
\end{aligned}
$$

The rest of the proof is analogous to what we did in the above and we omit it.

Proof of Theorem 4.2. Let $K$ be the convex conjugate of $\zeta$. That is

$$
K(\mu)=\sup _{f}\left(\int f d \mu-\zeta(f)\right),
$$

where the supremum is over all bounded continuous functions. We can obtain the upper bound large deviation estimate (2.1) for the family $\left\{Q_{N}\right\}$ when $C$ is compact by the same argument used in [14] (Lemma 11.3). To extend this to closed sets, we have to establish some kind of exponential tightness (see Stroock [12], page 46). More precisely, we need to show that for any large $\ell$ there exists a compact set $\mathbf{C}_{\ell}$ such that

$$
\lim _{\ell \rightarrow \infty} \limsup _{N \rightarrow \infty} \frac{1}{N^{d}} \log Q_{N}\left(C_{f}^{c}\right)=-\infty .
$$

Set $C_{\ell}=\{\mu:\|\mu\| \leqq \ell\}$, where $\|\mu\|$ denotes the total variation of $\mu$. $C_{\ell}$ is compact, and

$$
Q_{N}\left(C_{\ell}^{c}\right)=v_{N}\left(\frac{1}{N^{d}} \sum_{a}\left|x_{n}\right| \geqq \ell\right) \leqq e^{-\ell N^{d}} E^{v_{N}} \exp \left(\sum_{a}\left|x_{a}\right|\right) .
$$

Now (4.7) follows from (2.4).

So far we have shown the upper bound large deviation property for the family $\left\{Q_{N}\right\}$ with rate $K$. We will show in Lemma 5.4 that $K$ is equal to $J$ and $J$ is a rate function.

Lemma 4.4. $K=J$ and $J$ is a rate function.

Proof. Step 1. Let $f$ be a bounded measurable function. By Lusin's theorem, for any $\varepsilon>0$, there is a closed subset of torus $D_{\varepsilon}$ and a bounded continuous function $f_{\varepsilon}$ such that $\mu\left(D_{\varepsilon}^{c}\right)+\left|D_{\varepsilon}^{c}\right|<\varepsilon\left(|\cdot|\right.$ denotes the Haar measure on torus) and $\left.f_{\varepsilon}\right|_{D_{\varepsilon}}=\left.f\right|_{D_{\varepsilon}}$. From this we see that the supremum in (4.6) can be extended to all bounded measurable functions. 
Step 2. Let $d \mu$ be absolutely continuous with respect to $d \theta, g=d \mu / d \theta$. Set $g_{n}=(-n) \vee(g \wedge n)$ and $f_{n}=h^{\prime}\left(g_{n}\right)$. If $-n<g(x)<n$, then $g_{n}(x)=g(x)$ and $f_{n}(x)=$ $h^{\prime}(g(x))$. Therefore

$$
\int_{-n<g<n} h(g)=\int_{-n<g<n}\left[g f_{n}-\varphi\left(f_{n}\right)\right] \leqq \sup _{f} \int(g f-\varphi(f))=K(\mu) .
$$

On the other hand, it is easy to see $K(\mu) \leqq \int h(g)$. Thus

$$
K(\mu)=J(\mu) \text { if } \mu \ll d \theta .
$$

Final Step. We will finish the proof by showing $K(\mu)<\infty$ implies that $\mu \ll d \theta$. Since $K$ is convex and translation invariant, then

$$
K\left(k_{\varepsilon} * \mu\right) \leqq K(\mu)
$$

where $\left\{k_{\varepsilon}\right\}$ is an approximation to identity. Therefore, if $K(\mu)<\infty$, then by step 2 ,

$$
\sup _{\varepsilon>0} \int h\left(k_{\varepsilon} * \mu\right)<\infty \text {. }
$$

On the other hand $\lim _{|x| \rightarrow \infty}(h(x)) /|x|=+\infty$. In fact if $h\left(x_{n}\right) \leqq C x_{n}$ for some sequence $x_{n} \uparrow+\infty$, and some positive constant $C$, then $h(x) \leqq C x$ for large $x$, because $h$ is convex. But this implies that $\varphi(y)$ the convex conjugate of $h$ is infinite for $y>C$, and this is impossible. Therefore the sequence $\left\{k_{\varepsilon} * \mu\right\}$ is uniformly integrable, and this implies that $\mu$ is absolutely continuous.

$J$ is a rate function because

$$
\lim _{|x| \rightarrow \infty} \frac{h(x)}{|x|}=+\infty .
$$

\section{Hydrodynamic Limit}

Let $f_{N}^{0}$ be the initial density with respect to the Gibbs measure $v_{N}$. The measure $v_{N}$ is the Gibbs measure with periodic boundary conditions corresponding to the interaction field $F$, where $F$ is bounded, continuously differentiable and has bounded first derivatives. Then the evolution governed by $\mathscr{L}_{N}$ and starting from $f_{N}^{0}$ at time $t=0$, will give us a probability measure $Q_{N}$ on the space $C\left([0, \infty), \mathbf{R}^{N^{d}}\right)$. Let $\mathscr{M}_{\ell}$ denote the compact metric space (under weak convergence) of all signed measures of total variation at most $\ell$. For each $N$, and the set of trajectories $\left\{x_{a}(t): a \in S_{N}^{d}\right\}$ we define

$$
\mu(t)=\frac{1}{N^{d}} \sum_{a \in S_{N}^{d}} x_{a}(t) \delta_{a / N}
$$

in the space $\cup, \mathscr{M}_{\ell}$. This, as a function of $t \in\left[0, t_{0}\right]$, is an element of $\Omega=$ $\cup_{\ell} C\left(\left[0, t_{0}\right], \mathscr{M}_{\ell}\right)$, where $C\left(\left[0, t_{0}\right], \mathscr{M}_{\ell}\right)$ is the space of weakly continuous maps from $\left[0, t_{0}\right]$ into $\mathscr{M}_{\ell}$. Then (5.1) introduces a probability measure $\hat{Q}_{N}$ on the space $\Omega$. It is not hard to see that the sequence $\hat{Q}_{N}$ is tight. This follows Lemmas 6.1 and 6.2 in [4] adapted to our setting. Let $Q$ be any limit point of the sequence $Q_{N}$. Then 
Theorem 5.1. $Q$ is concentrated on the single path $\mu(t, d \theta)=m(t, \theta) d \theta$ that is the unique weak solution of

$$
\frac{\partial m}{\partial t}=\frac{1}{2} \Delta h^{\prime}(m), \quad m(0, \theta)=m_{0}(\theta)
$$

satisfying the estimates

$$
\begin{gathered}
\int h(m(t, \theta)) d \theta \leqq C, \\
\frac{1}{2} \iint_{0}^{\infty}\left|\nabla h^{\prime}(m(s, \theta))\right|^{2} d s d \theta \leqq C .
\end{gathered}
$$

for all $t \geqq 0$.

Proof. Since the entropy is decreasing, we have

$$
\frac{1}{N^{d}} \int f_{N}^{t} \log f_{N}^{t} d v_{N} \leqq C
$$

for all $t \geqq 0$. Therefore, by Theorem 4.1 we have $\mu(t, d \theta)=m(t, \theta) d \theta$ and

$$
E^{Q} \int h(m(t, \theta)) d \theta \leqq C .
$$

This and the uniqueness result (Theorem 5.2) imply (5.3). The inequality (3.16) allows us to follow the proof of Theorem 5.1 and Lemma 6.6 in [4] to conclude (5.4). The uniqueness of the solution of (5.2) under the conditions (5.3) and (5.4) will be shown in Theorem 5.2 below.

We now turn to the proof of (5.2). Let $J$ be a smooth test function. Consider the functional

$$
\begin{aligned}
V(t, N, \varepsilon, k ; \mu)= & \int J(\theta) \mu(t, d \theta)-\int J(\theta) \mu(0, d \theta) \\
& -\frac{1}{2 N^{d}} \int_{0}^{t} \sum_{a \in S_{N}^{d}} \Delta J(a / N) \psi_{k}{ }^{\circ} h^{\prime}\left(m_{T_{N_{\varepsilon}}}\left(\tau_{a} \underline{x}(s)\right)\right) d s .
\end{aligned}
$$

By Itô's formula

$$
\begin{aligned}
& \frac{1}{N^{d}} \sum_{a} J(a / N) x_{a}(t)-\frac{1}{N^{d}} \sum_{a} J(a / N) x_{a}(0) \\
& \quad=\frac{1}{2 N^{d}} \sum_{a} \int_{0}^{t} \Delta J(a / N) G_{0}\left(\tau_{a} \underline{x}(s)\right) d s+M_{N}(t)+r_{N},
\end{aligned}
$$

where $M_{N}$ is a Martingale and $\lim _{N \rightarrow \infty} r_{N}=0$ in probability (see the proof of Lemma 3.4.(b) for the way we treated the unboundedness of $\left.G_{0}\right) . r_{N}$ is an error coming from replacing $\left(1 / N^{2}\right) \sum_{j}\left[J\left(a / N+e_{j} / N\right)+J\left(a / N-e_{j} / N\right)-2 J(a / N)\right]$ with $\Delta J(a / N)$ in Itô's formula. An explicit calculation shows $\lim _{N \rightarrow \infty} E^{Q_{N}}\left|M_{N}(t)\right|^{2}=0$. This and Theorem 3.1 imply

$$
\lim _{k \rightarrow \infty} \limsup _{\varepsilon \rightarrow 0} \limsup _{N \rightarrow \infty} E^{Q_{N}}|V(t, N, \varepsilon, k ; \mu)|=0 .
$$


Therefore

$$
\lim _{k \rightarrow \infty} E^{Q}|V(t, k ; \mu)|=0
$$

where

$$
\begin{aligned}
V(t, k ; \mu)= & \int J(\theta) \mu(t, d \theta)-\int J(\theta) \mu(0, d \theta) \\
& -\frac{1}{2} \int_{0}^{t} \int \Delta J(\theta) \psi_{k} \circ h^{\prime}(m(s, \theta)) d \theta d s .
\end{aligned}
$$

This is because when $a / N$ is close $\theta_{0}$, then $m_{T_{N \varepsilon}}\left(\tau_{a} \underline{x}(s)\right)$ is almost the average of $\mu(s, d \theta)$ over the cube of side $2 \varepsilon$ and center $\theta_{0}$ divided by its volume. This converges to $m\left(s, \theta_{0}\right)$ as $\varepsilon$ tends to zero.

Finally, using Lemma 2.21 and Theorem 4.1 we can pass to the limit as $k \rightarrow \infty$ and yield

$$
E^{Q}\left|\int J(\theta) \mu(t, d \theta)-\int J(\theta) \mu(0, d \theta)-\frac{1}{2} \int_{0}^{t} \int \Delta J(\theta) h^{\prime}(m(s, \theta)) d s d \theta\right|=0
$$

and this completes the proof.

Theorem 5.2. The weak solution of (5.2) is unique within the class of functions $m(t, \theta)$ satisfying (5.3) and (5.4).

Proof. Consider the nonlinear operator $A(m)=-\Delta h^{\prime}(m)$ with

$$
\mathscr{D}(A)=\left\{m \in H^{-1} \cap L^{1}: h^{\prime}(m) \in H^{1}\right\} .
$$

The operator $A$ is maximal and monotone in the sense that

$$
\left\langle m_{1}-m_{2}, A m_{1}-A m_{2}\right\rangle_{H^{-1}} \geqq 0
$$

for $m_{1}, m_{2} \in H^{-1}$, and the graph of $A$ as a subset of $H^{-1} \times H^{-1}$ is maximal within the graph monotone operators (see Barbu [1] for more details). Thus we have uniqueness of the solution of (5.2) in the $H^{-1}$-sense (this follows from the monotonicity property). For the rest of the proof, we verify that if the weak solution of (5.2) satisfies (5.4), then this solution is also a strong solution in the space of $\mathrm{H}^{-1}$.

Let $v(t)=\int_{0}^{t} \nabla h^{\prime}(m(s)) d s$. Then $v$ is absolutely continuous as a function from $[0, \infty)$ into $L^{2}$, and $v^{\prime}(t)=\nabla h^{\prime}(m(t))$ for almost all $t$. Since $m(t, \cdot)$ is a weak solution of (5.2), we have $\frac{1}{2} \operatorname{div} v(t)=m(t)-m(0)$ in distribution. Therefore $\|m(t)-m(s)\|_{H^{-1}}^{2} \leqq$ $(d / 4)\|v(t)-v(s)\|_{L^{2}}^{2}$ and this implies that $m:[0, \infty] \rightarrow H^{-1}$ is absolutely continuous and $d m / d t=\frac{1}{2} \Delta h^{\prime}(m)$ for almost all $t$, in the $H^{-1}$-sense.

When $h^{\prime}$ is not strictly increasing, the solutions of (5.2) might be singular. If, however, $m(0)=m_{0}$ is in a single phase (for example $m_{0}$ is in a region on which $h^{\prime \prime}$ is uniformly positive), then $m(t)$ will stay in that phase for all $t \geqq 0$. This is evident in the following theorem.

Theorem 5.3. (Maximum Principle). Suppose that $m_{1}(t)$ and $m_{2}(t)$ are two solutions of (5.2) such that $m_{1}(0), m_{2}(0) \in L^{\infty}$ and

$$
m_{1}(0, \theta) \leqq m_{2}(0, \theta)
$$


for almost all $\theta$, then

$$
m_{1}(t, \theta) \leqq m_{2}(t, \theta)
$$

for all $t$ and almost all $\theta$.

Proof. Let $A^{\varepsilon} u=-\Delta \lambda^{\varepsilon}(u)$, where

$$
\lambda^{\varepsilon}(x)=\frac{1}{\varepsilon} \int h^{\prime}(y) k\left(\frac{x-y}{\varepsilon}\right) d y+\varepsilon x,
$$

where $k: \mathbf{R} \rightarrow \mathbf{R}^{+}$is a smooth function with support in the interval $[-1,1]$. Then $\lambda^{\varepsilon}(\cdot)$ is smooth with $(d / d x) \lambda^{\varepsilon}(x) \geqq \varepsilon$ for all $x$. We also have

$$
\lambda^{\varepsilon}(x) \rightarrow h^{\prime}(x)
$$

uniformly in bounded subsets of $\mathbf{R}$.

Let $m_{i}^{\varepsilon}(t)$ be the unique solution (in $H^{-1}$-sense) of $(d m / d t)+\frac{1}{2} A^{\varepsilon} m=0$ such that $m_{i}^{\varepsilon}(0)=m_{i}(0)$ for $i=1$ or 2 . We then have

$$
\begin{aligned}
\frac{d}{d t}\left\|m_{i}(t)-m_{i}^{\varepsilon}(t)\right\|_{H^{-1}}^{2} & =\left\langle m_{i}(t)-m_{i}^{\varepsilon}(t), A\left(m_{i}(t)\right)-A^{\varepsilon}\left(m_{i}^{\varepsilon}(t)\right)\right\rangle_{H^{-1}} \\
& =\left\langle m_{i}(t)-m_{i}^{\varepsilon}(t), h^{\prime}\left(m_{i}(t)\right)-\lambda^{\varepsilon}\left(m_{i}^{\varepsilon}(t)\right)\right\rangle_{L^{2}}
\end{aligned}
$$

On the other hand, we can use the maximum principle for solutions $m_{i}^{\varepsilon}$ to obtain

$$
m_{1}^{\varepsilon}(t, \theta) \leqq m_{2}^{\varepsilon}(t, \theta)
$$

for all $t$ and $\theta$. We also have $\sup _{t, \theta}\left|m_{i}^{\varepsilon}(t, \theta)\right|<\infty$ for $i=1,2$. Therefore, by (5.6),

$$
\lim _{\varepsilon \rightarrow 0} \sup _{t} \| h^{\prime}\left(m_{i}^{\varepsilon}(t)\right)-\lambda^{\varepsilon}\left(m_{i}^{\varepsilon}(t) \|_{L^{2}}=0 .\right.
$$

This allows us to replace $\lambda^{\varepsilon}\left(m_{i}^{\varepsilon}(t)\right)$ with $h^{\prime}\left(m_{i}^{\varepsilon}(t)\right)$ in (5.7). So

$$
\limsup _{\varepsilon \rightarrow 0} \sup _{t} \frac{d}{d t}\left\|m_{i}^{\varepsilon}(t)-m_{i}(t)\right\|_{H^{-1}}^{2} \leqq 0
$$

for $i=1,2$. Therefore $m_{i}^{\varepsilon}(t)$ converges to $m_{i}(t)$ in $H^{-1}$, for all $t$. This and (5.8) imply (5.5).

It is of interest to study the asymptotic behavior of the solution $m(t)$ when $t$ goes to infinity. For an account of this problem, see [5], Lecture 26. Here, we confine ourselves to the case $m_{0} \in L^{\infty}$, and we show that the limit of $m(t)$ as $t$ goes to infinity, exists.

Theorem 5.4. If $m_{0} \in L^{\infty}$, then $\lim _{t \rightarrow \infty} m(t)=m(\infty)$ exists in $H^{-1}$, and we have

$$
h^{\prime}\left(m\left(\infty, \theta_{0}\right)\right)=h^{\prime}\left(\int m_{0}(\theta) d \theta\right)
$$

for almost all $\theta_{0}$.

Proof. Since $m_{0} \in L^{\infty}$, we have $\sup \|m(t)\|_{L^{\infty}}<\infty$. Therefore $\{m(t): t \geqq 0\}$ is a precompact subset of $H^{-1}$. Taking into account the condition (5.4), we may choose 
a sequence of $t_{n} \uparrow \infty$, such that

$$
\begin{gathered}
m\left(t_{n}\right) \rightarrow m(\infty) \text { in } H^{-1}, \\
\nabla h^{\prime}\left(m\left(t_{n}\right)\right) \rightarrow 0 \text { in } L^{2},
\end{gathered}
$$

for some $m(\infty) \in H^{-1}$. Since $\int m\left(t_{n}, \theta\right) d \theta=\int m_{0}(\theta) d \theta$, we have $\int m(\infty, \theta) d \theta=\int m_{0}(\theta) d \theta$ and (5.11) guarantees that we also have (5.9).

Finally $\|m(t)-m(\infty)\|_{H^{-1}}$ is nonincreasing in $t$ (because of $(5.9), v(t)=m(\infty)$ is a steady solution of $(5.2))$, thus $\lim m(t)=m(\infty)$ in $H^{-1}$.

Acknowledgement. I am very grateful to Professor Srinivasa R. S. Varadhan for suggesting this problem and for his help and encouragement in this work.

\section{References}

1. Barbu, V.: Nonlinear semigroups and differential equations in banach spaces. Noordhoff 1976

2. Ellis, R. S.: Entropy, large deviations and statistical mechanics. Grundleheren der Mathematischen Wissenschaften vol. 271. Berlin, Heidelberg, New York: Springer 1985

3. Fritz, J.: On the hydrodynamic limit of a Ginzburg-Landau lattice model. Prob. Th. Rel. Fields 81, 291-318 (1989)

4. Guo, M. Z., Papanicoulaou, G. C., Varadhan, S. R. S.: Nonlinear diffusion limit for a system with nearest neighbor interactions. Commun. Math. Phys. 118, 31-59 (1988)

5. Haraux, A.: Nonlinear evolution equations, global behavior of solutions. Lecture Notes in Mathematics, vol. 841. Berlin, Heidelberg, New York: Springer 1981

6. Kipnis, C., Olla, S., Varadhan, S. R. S.: Hydrodynamics and large deviations for simple exclusion process. Commun. Pure Appl. Math. (to appear 1989)

7. Ladyženskaja, O. A., Solonnikov, V. A., Ural'ceva, N. N.: Linear and quasilinear equations of parabolic type. Transl. Math. Monographs, Vol. 23, Providence, RI: Am. Math. Soc. 1968

8. Lifshitz, E. M., Pitaevskii, L. P.: Course on theoretical physics, vol. 10. Physical kinetics. Oxford: Pergamon Press 1981

9. Olla, S.: Large deviations for Gibbs random fields. Prob. Th. Rel. Fields 77, 343-357 (1988)

10. Preston, C. J.: Random Fields. Lecture Notes in Mathematics, vol. 534. Berlin, Heidelberg, New York: Springer 1976

11. Spohn, H.: Equilibrium fluctuations for some stochastic particle systems. In: Fritz, J., Jaffe, A., Szasz, D. (eds.). Statistical physics and dynamical systems. pp. 67-81. Basel-Boston: Birkhäuser (1985)

12. Stroock, D. W.: An introduction to the theory of large deviations. Berlin, Heidelberg, New York: Springer 1984

13. Varadhan, S. R. S.: Asymptotic probabilities and differential equations. Commun. Pure Appl. Math. 19, 261-286 (1966)

14. Varadhan, S. R. S.: Large deviations and applications. CBMS-NSF Regional Conference Series in Applied Mathematics, Vol. 46, SIAM (1984)

15. Varadhan, S. R. S.: On the derivation of conservation laws for stochastic dynamics, preprint

Communicated by J. L. Lebowitz

Received June 22, 1989; in revised form October 12, 1989 